Article

\title{
Proteomic Investigation of S-Nitrosylated Proteins During NO-Induced Adventitious Rooting of Cucumber
}

\author{
Lijuan Niu, Jihua Yu*, Weibiao Liao®, Jianming Xie, Jian Yu, Jian Lv, Xuemei Xiao, Linli Hu and \\ Yue Wu \\ College of Horticulture, Gansu Agricultural University, Lanzhou 730070, China; niulj0508@163.com (L.N.); \\ liaowb@gsau.edu.cn (W.L.); xiejianming@gsau.edu.cn (J.X.); Yjian0515@163.com (J.Y.); lvjian@gsau.edu.cn (J.L.); \\ xiaoxm@gsau.edu.cn (X.X.); hull@gsau.edu.cn (L.H.); wuyue_gsau@163.com (Y.W.) \\ * Correspondence: yujihuagg@163.com; Tel.: +86-13609308343
}

Received: 10 September 2019; Accepted: 23 October 2019; Published: 28 October 2019

check for updates

\begin{abstract}
Nitric oxide (NO) acts an essential signaling molecule that is involved in regulating various physiological and biochemical processes in plants. However, whether $S$-nitrosylation is a crucial molecular mechanism of $\mathrm{NO}$ is still largely unknown. In this study, $50 \mu \mathrm{M} S$-nitrosoglutathione (GSNO) treatment was found to have a maximum biological effect on promoting adventitious rooting in cucumber. Meanwhile, removal of endogenous NO significantly inhibited the development of adventitious roots implying that $\mathrm{NO}$ is responsible for promoting the process of adventitious rooting. Moreover, application of GSNO resulted in an increase of intracellular S-nitrosothiol (SNO) levels and endogenous NO production, while decreasing the $S$-nitrosoglutathione reductase (GSNOR) activity during adventitious rooting, implicating that $S$-nitrosylation might be involved in NO-induced adventitious rooting in cucumber. Furthermore, the identification of $S$-nitrosylated proteins was performed utilizing the liquid chromatography/mass spectrometry/mass spectrometry (LC-MS/MS) and biotin-switch technique during the development of adventitious rooting. Among these proteins, the activities and S-nitrosylated level of glyceraldehyde-3-phosphate dehydrogenase (GAPDH), tubulin alpha chain (TUA), and glutathione reductase (GR) were further analyzed as NO direct targets. Our results indicated that NO might enhance the $S$-nitrosylation level of GAPDH and GR, and was found to subsequently reduce these activities and transcriptional levels. Conversely, $S$-nitrosylation of TUA increased the expression level of TUA. The results implied that S-nitrosylation of key proteins seems to regulate various pathways through differential $S$-nitrosylation during adventitious rooting. Collectively, these results suggest that $S$-nitrosylation could be involved in NO-induced adventitious rooting, and they also provide fundamental evidence for the molecular mechanism of NO signaling during adventitious rooting in cucumber explants.
\end{abstract}

Keywords: Nitric oxide; S-nitrosylation; adventitious root development

\section{Introduction}

Free radical nitric oxide (NO) is generated via non-enzymatic [1] and enzymatic pathways [2,3] in plants. As a multifunctional physiological regulator, NO has been shown to be involved in every aspect of plant growth and every developmental process in plants [4]. Furthermore, an increasing body of evidence has indicated that NO could play an essential role in response to various abiotic stresses [5-7].

Previous studies suggested that NO could exert its effects depending on the cyclic guanosine monophosphate (cGMP) signaling pathway $[8,9]$. For example, NO could promote the adventitious rooting of marigold through the cGMP-dependent pathway [10]. 
Additionally, the emerging picture is that $\mathrm{NO}$ also could operate biological functions through protein S-nitrosylation which is a NO-dependent posttranslational modification (PTM) [11,12]. It has been shown that $\mathrm{NO}$ groups could be covalently bound to cysteine (Cys) residues of target proteins, resulting in the formation of $S$-nitrosothiols during $S$-nitrosylation [11,13]. At present, increasing evidence demonstrates that $S$-nitrosylation might be involved in processes for regulating the growth, development, and stress responses in plants [14-16].

In general, proteomics deals with the large-scale determination of gene and cellular function directly at the protein level [17]. Recently, global protein S-nitrosylation has been identified using the proteomic approach. According to Hu et al. [18], more than $2200 S$-nitrosylated proteins have been identified in mammals and plants. In the present work, several $S$-nitrosylated proteins have been identified using proteomic analyses in different plants. For example, Lindermayr et al. [19] identified proteins, which, when treated with NO, were involved in various pathways such as cytoskeleton organization, metabolic processes, redox homeostasis, as well as cellular signaling transduction. Moreover, Morisse et al. [20] identified 492 S-nitrosylated proteins and 392 sites in chlamydomonas reinhardtii cells, which were treated with S-nitrosoglutathione (GSNO). Moreover, 926 proteins that undergo nitrosylation have been identified in Arabidopsis [18]. Certain NO target proteins have been pointed out as important for regulating physiological and pathological cellular processes through proteomic and transcriptomic analyses [21-23]. Although the identification research on S-nitrosylation is increasing, the mechanism of $S$-nitrosylation during root development remains unclear. The aim of this study was to identify possible candidates for $S$-nitrosylation during adventitious rooting to reveal the biological function of NO at the protein level in plants. Therefore, we conducted this experiment to detect and identify the $S$-nitrosylated proteins during NO-induced adventitious rooting in cucumber explants. The objective of this study was to decipher the novel role of protein S-nitrosylation in the process of adventitious root development in order to further improve our understanding of NO signaling transduction in molecular mechanisms.

\section{Results}

\subsection{Effect of Exogenous S-Nitrosoglutathione (GSNO) on Adventitious Rooting in Cucumber}

In order to access the effects of exogenous GSNO on adventitious root development of cucumber, explants were cultivated with different concentrations of $\operatorname{GSNO}(0,0.1,1,10,50$ and $100 \mu \mathrm{M})$. As shown in Figure 1, there is no significant difference between the control and $0.1 \mu \mathrm{M}$ GSNO. Meanwhile, lower concentrations of GSNO $(1,10$, and $50 \mu \mathrm{M})$ significantly promoted the development of adventitious root. However, a higher dose of GSNO $(100 \mu \mathrm{M})$ obviously decreased the number and length of adventitious roots, indicating exogenous GSNO could have a concentration-dependent effect on adventitious rooting. Moreover, the root number and length of $50 \mu \mathrm{M}$ GSNO-treated explants increased by $92 \%$ and $280.60 \%$, respectively, when compared with the control (Figure 1 ). These results revealed that $50 \mu \mathrm{M}$ GSNO had the maximum biological effect on adventitious rooting. Therefore, $50 \mu \mathrm{M}$ GSNO was used for the following experiments. 


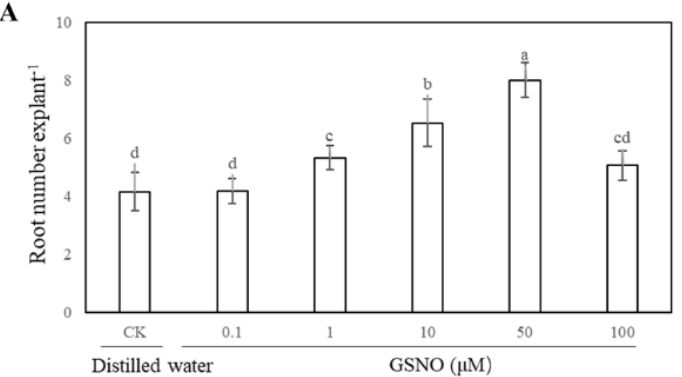

B

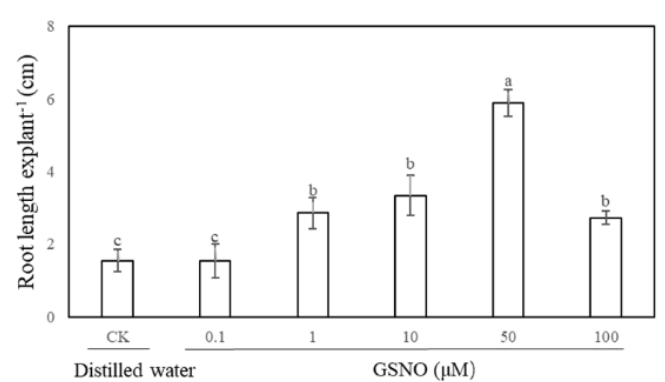

C

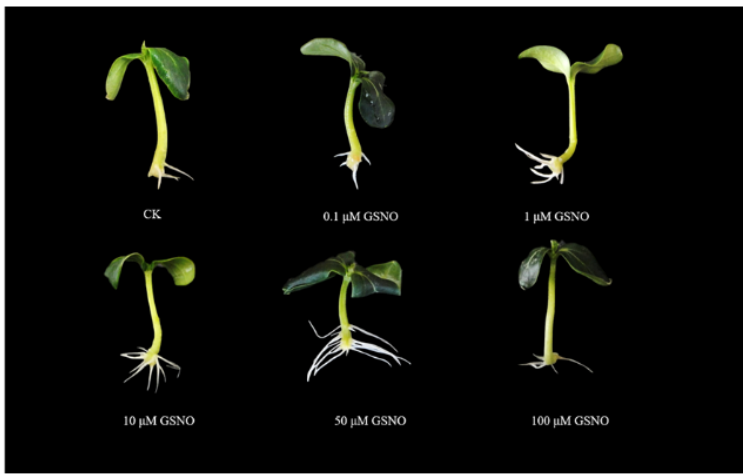

Figure 1. Effect of different concentrations of $S$-nitrosoglutathione (GSNO) on adventitious root development in cucumber explants. The primary roots were removed from hypocotyl of 5-day-old seedlings. Explants were incubated for 5 days with different concentrations of GSNO. The numbers (A) and root length (B) of adventitious roots were expressed as mean $\pm \mathrm{SE}(n=3)$. Ten explants were used per replicate. Photographs $(\mathbf{C})$ were taken after five days of the treatments indicated. Bars with different lowercase letters were significantly different by Duncan's multiple range test $(p<0.05)$. Bars with different lowercase letters were significantly different by Duncan's multiple range test.

\subsection{Effect of Nitric Oxide (NO) Scavenger on Adventitious Rooting in Cucumber}

In order to further confirm the effect of $\mathrm{NO}$ on adventitious rooting, $\mathrm{NO}$ scavenger 2-(4-carboxy-2-phenyl)-4, 4, 5, 5-tetramethylimidazoline-1-oxyl-3-oxide (cPTIO), and a normal product of $\mathrm{NO}$ decomposition, $\mathrm{NaNO}_{3}$ were applied in our research. Figure 2 showed that application of cPTIO alone clearly inhibited the adventitious root development. $\mathrm{NaNO}_{3}$ treatment as a control for $\mathrm{NO}$ decomposition had no effect on adventitious root development. However, GSNO + cPTIO treatment significantly reversed the inhibitive effect of NO scavengers (Figure 2). These results indicate that NO is responsible for the development of adventitious root in cucumber explants. 

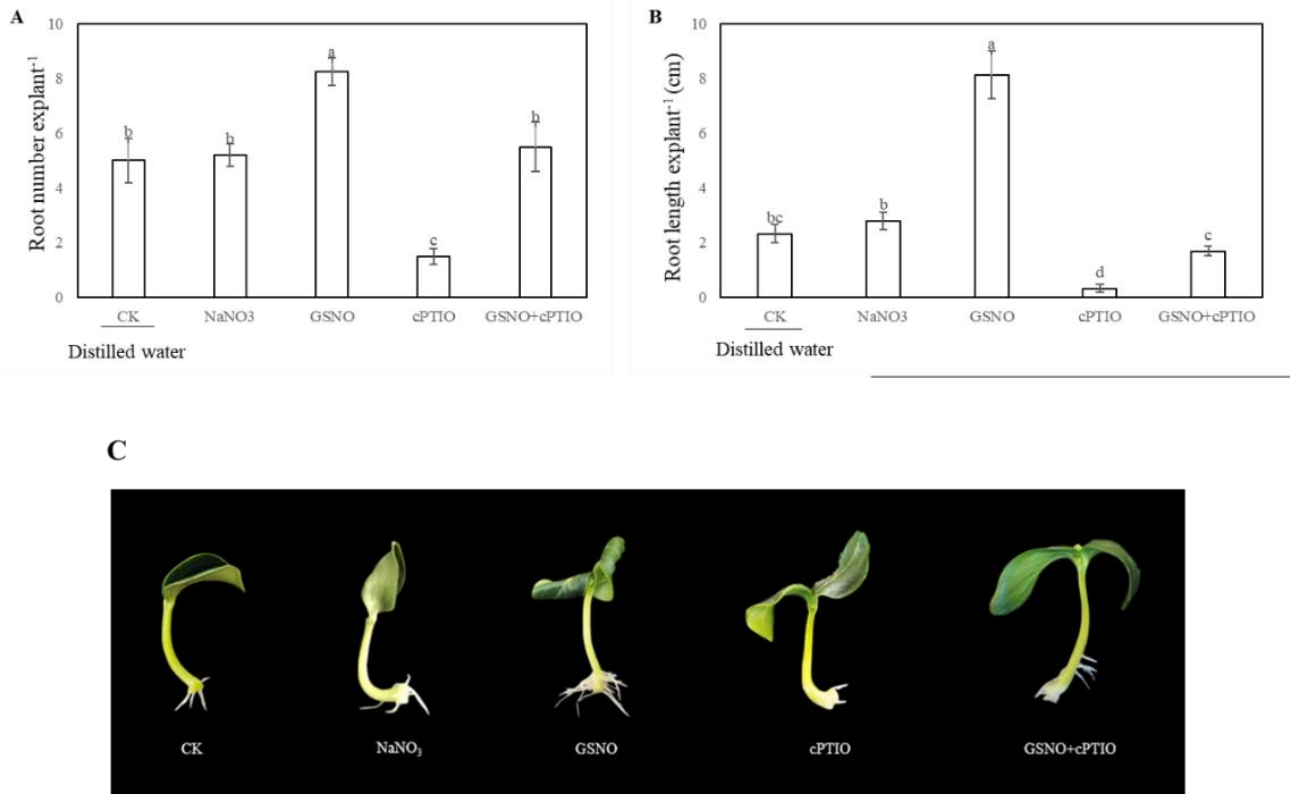

Figure 2. Effect of NO scavenger 2-(4-carboxy-2-phenyl)-4, 4, 5, 5-tetramethylimidazoline-1-oxyl-3-oxide (cPTIO) on adventitious root development in cucumber explants. The primary roots were removed from 5-day-old seedlings. Explants were then incubated for 5 days with distilled water (CK) or $100 \mu \mathrm{M}$ sodium nitrate $\left(\mathrm{NaNO}_{3}\right), 50 \mu \mathrm{M}$ GSNO, $200 \mu \mathrm{M}$ cPTIO, or $50 \mu \mathrm{M}$ GSNO + $200 \mu \mathrm{M}$ cPTIO. The numbers (A) and root length $(\mathbf{B})$ of adventitious roots were expressed as mean $\pm \mathrm{SE}(n=3)$. Ten explants were used per replicate. Photographs $(\mathbf{C})$ were taken after five days of the treatments indicated. Bars with different lowercase letters were significantly different by Duncan's multiple range test $(p<0.05)$. Bars with different lowercase letters were significantly different by Duncan's multiple range test.

2.3. Effect of GSNO on the Levels of Total S-Nitrosothiol (SNO), and S-Nitrosoglutathione Reductase (GSNOR) Activity and Endogenous NO Level During the Development of Adventitious Roots in Cucumber

To further elucidate whether $S$-nitrosylation was involved in the process of adventitious rooting, the level of endogenous S-nitrosothiol (SNO) was tested during adventitious rooting (Figure 3A). As shown in Figure 3A, during adventitious rooting, treatment with GSNO strikingly elevated the endogenous SNO level. At $6 \mathrm{~h}$, nitroso groups with GSNO treatment reached the maximum value and were significantly higher than that of cPTIO treatment. On the contrary, lower S-nitrosoglutathione reductase (GSNOR) activity was found in GSNO treatment relative to that of control or cPTIO treatment at $6 \mathrm{~h}$ (Figure 3B). Additionally, application of GSNO treatment significantly enhanced the fluorescent intensity of NO production in cucumber hypocotyl. Meanwhile, there was no significant difference between distilled water $(\mathrm{CK})$ treatment and sodium nitrate $\left(\mathrm{NaNO}_{3}\right)$ treatment (Figure $\left.3 \mathrm{C}, \mathrm{D}\right)$. However, the production of endogenous NO was remarkably reduced in hypocotyl after NO scavenger treatment (Figure 3). 

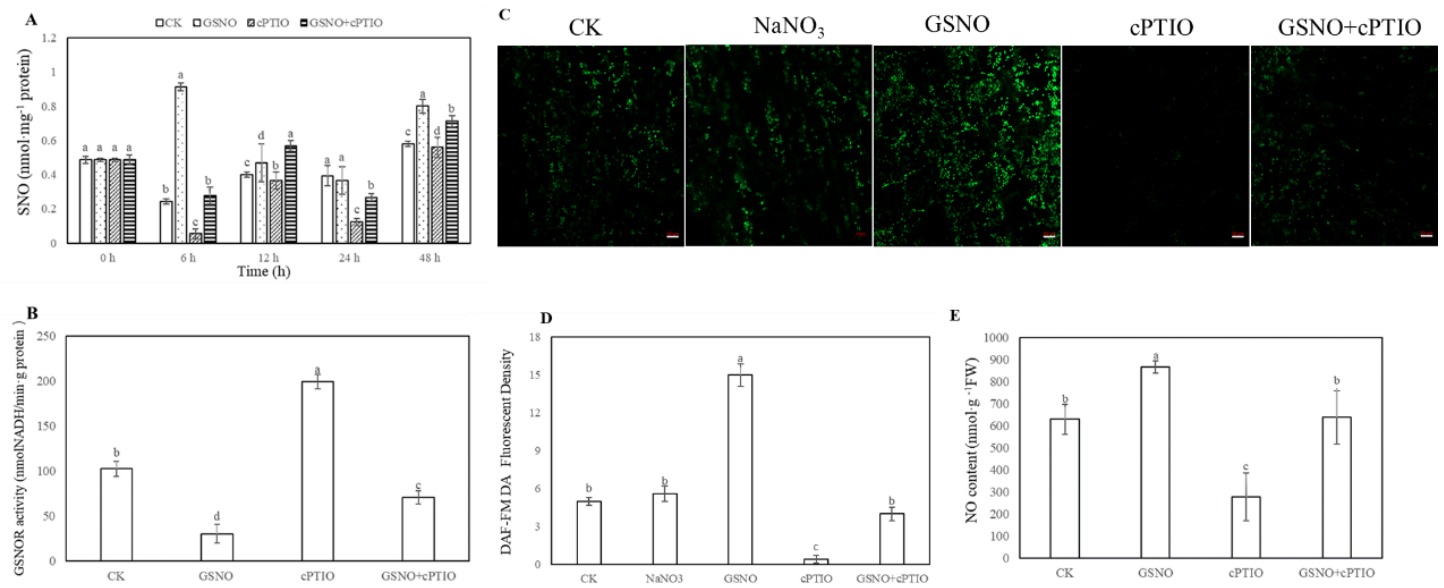

Figure 3. Effect of GSNO on the levels of total S-nitrosothiol (SNO) (A), S-nitrosoglutathione reductase (GSNOR) activity (B) and endogenous NO level (C, D, E) during the development of adventitious roots in cucumber. Explants were incubated with distilled water (CK) or $100 \mu \mathrm{M} \mathrm{NaNO}, 200 \mu \mathrm{M}$ cPTIO, $50 \mu \mathrm{M}$ GSNO, or $50 \mu \mathrm{M}$ GSNO $+200 \mu \mathrm{M}$ cPTIO. The levels of total SNO (A) were determined during adventitious rooting. GSNOR activity $(\mathbf{B})$ and endogenous NO levels $(\mathbf{C}, \mathbf{E})$ in cucumbers were detected after $6 \mathrm{~h}$ of treatment. 4-amino-5-methylamino-2', $7^{\prime}$ - di aminofluoresceindiacetate (DAF-FM DA) was utilized to detect endogenous NO of a longitudinal section from the tip of the hypocotyls. Changes in fluorescence intensity of $\mathrm{NO}(\mathrm{C})$ were monitored by fluorescence microscopy after $6 \mathrm{~h}$. The DAF-FM DA fluorescence density of endogenous NO (D) was analyzed by ImageJ software. Bars with different lowercase letters were significantly different by Duncan's multiple range test $(p<0.05)$. Bars with different lowercase letters were significantly different by Duncan's multiple range test.

\subsection{Identification of S-Nitrosylated Proteins During NO-Induced Adventitious Rooting in Cucumber}

In order to further identify whether there exist possible candidates for $S$-nitrosylation during NO-induced adventitious rooting in cucumber explants, biotin switch detection and liquid chromatography/mass spectrometry/mass spectrometry (LC-MS/MS) were performed (Figure 4). As shown in Figure 4A,B, GSNO treatment obviously increased nitrosylation of proteins during adventitious rooting of cucumber, when compared to those of the control treatment. However, cPTIO treatment remarkably inhibited potential candidates for $S$-nitrosylation. Moreover, our results indicated that 167 proteins were identified from control, GSNO treatment, and GSNO + cPTIO treatment (Table 1). These identified proteins might be involved in various processes during adventitious rooting such as carbon and energy metabolism, photosynthesis, transcription and translation, and so on (Figure 4C). During adventitious rooting, approximately $40 \%$ were found to function in carbon and energy metabolism, $25.5 \%$ in the process of genetic information, and $8.5 \%$ in the growth and development process (Figure 4C). Additionally, identified proteins were found to function related to redox homeostasis, signaling transduction, and hormone response, about $9.7 \%, 3.0 \%$, and $1.8 \%$, respectively (Figure $4 \mathrm{C}$ ). Among these proteins, three and 48 proteins were identified from the control and GSNO treatment, respectively (Figure 4D-F). As shown in Figure 4D, 114 proteins are common to both the control and GSNO treatment. These results implied that $S$-nitrosylation might be involved in NO-induced adventitious rooting in cucumber. 

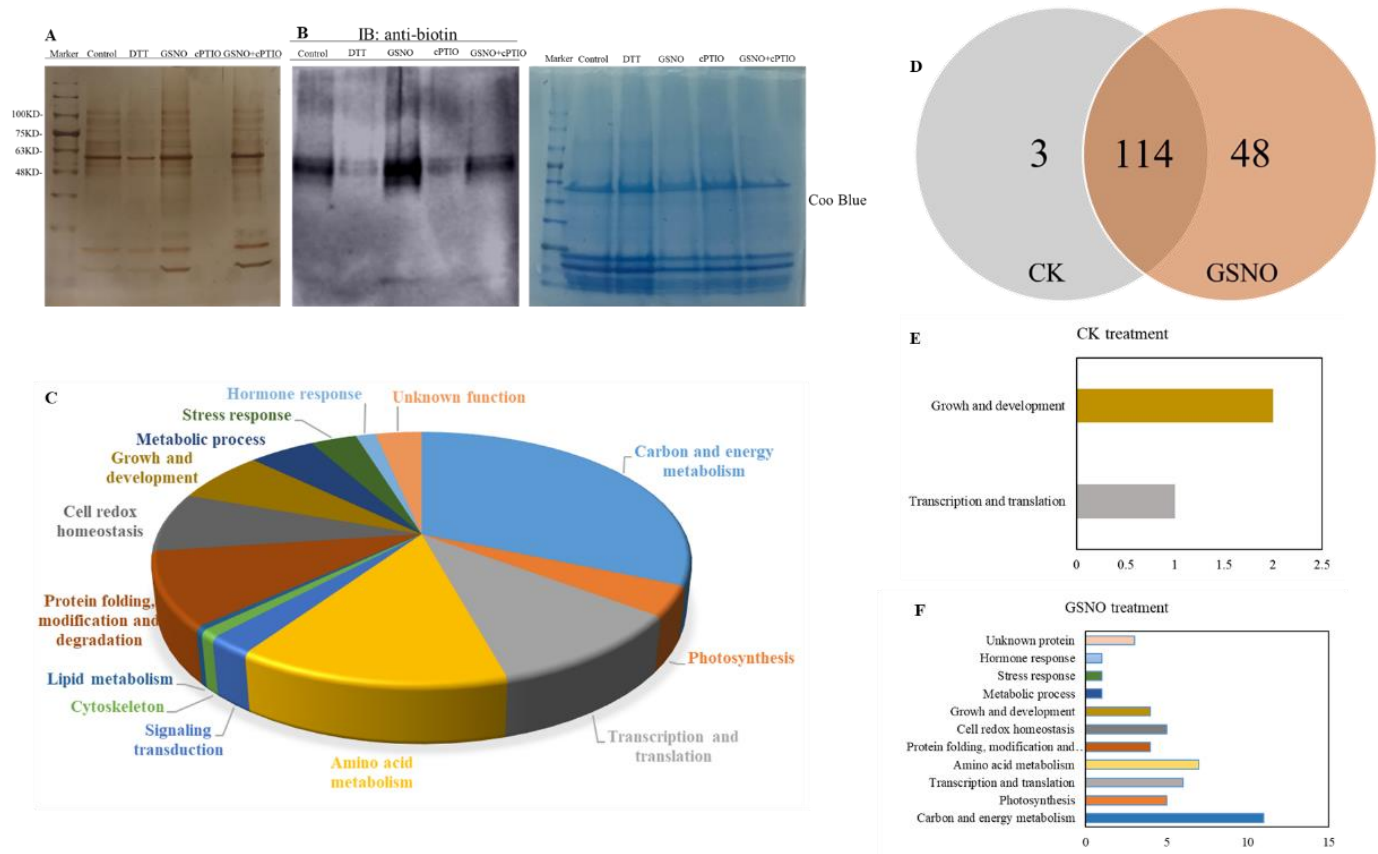

Figure 4. Identification of S-nitrosylated proteins during the development of adventitious rooting. Total S-nitrosylated proteins were detected through liquid chromatography/mass spectrometry/mass spectrometry (LC-MS/MS) and the western blotting method after explants were incubated with distilled water (CK) or $50 \mu \mathrm{M}$ GSNO, $200 \mu \mathrm{M}$ cPTIO, or $50 \mu \mathrm{M}$ GSNO + $200 \mu \mathrm{M}$ cPTIO for $6 \mathrm{~h}(\mathrm{~A}, \mathbf{B})$. Functional categorization of $S$-nitrosylated proteins from CK, GSNO and GSNO + cPTIO treatment $(\mathrm{C})$. The number of $S$-nitrosylated proteins in CK and GSNO treated explants (D). Functional categorization of $S$-nitrosylated proteins from CK treatment alone (E). Functional categorization of $S$-nitrosylated proteins from GSNO treatment alone $(\mathbf{F})$. 
Table 1. S-nitrosylated proteins identified from the control, S-nitrosoglutathione (GSNO), and GSNO + 2-(4-carboxy-2-phenyl)-4, 4, 5, 5-tetramethylimidazoline-1-oxyl-3-oxide (cPTIO) treatment during adventitious rooting of cucumber seedlings.

\begin{tabular}{|c|c|c|c|}
\hline Accession Number & Protein Name & Mol Mass & Peptide Sequence \\
\hline A0A0A0K9P5 & 11S globulin subunit beta-like & $54 \mathrm{kDa}$ & SSLLAFLC ${ }^{11}$ LAVFING NGFEETVC ${ }^{299}$ TLRLKHN \\
\hline A0A0A0K674 & $26 S$ protease regulatory subunit 7 & $47 \mathrm{kDa}$ & $\begin{array}{c}\text { AKKVNDLC }{ }^{56} \text { GIKESDT QPLQVARC }^{91} \text { TKIINPN } \\
\text { MARSKKAC }{ }^{263} \text { IVFFDEV }\end{array}$ \\
\hline $\mathrm{A} 0 \mathrm{~A} 0 \mathrm{~A} 0 \mathrm{~K} 3 \mathrm{C} 4$ & $\begin{array}{c}26 \mathrm{~S} \text { proteasome non-ATPase regulatory subunit } 2 \\
\text { homolog }\end{array}$ & $98 \mathrm{kDa}$ & GLIYLGSC ${ }^{539}$ NEEVAQA \\
\hline A0A0A0LQ32 & 4-alpha-glucanotransferase & $64 \mathrm{kDa}$ & YSGQDANC ${ }^{140}$ GNTLLIS \\
\hline A0A0A0KAJ9 & 60 S ribosomal protein L3 & $44 \mathrm{kDa}$ & KDDATKPC ${ }^{41}$ RLTAFLG \\
\hline A0A0A0KXM8 & 6-phosphogluconate dehydrogenase, decarboxylating & $53 \mathrm{kDa}$ & AYLEKGDC ${ }^{103}$ IIDGGNE \\
\hline Q08375 & Acetyl-CoA acyltransferase (3-ketoacyl-coa thiolase) & $48 \mathrm{kDa}$ & $\begin{array}{l}\text { SIENAQNC }{ }^{191} \text { LLPMGVT FASQFVYC }^{370} \text { RNKLGLD } \\
\text { LGATGARC }^{401} \text { VATLLHE AVFERGDC } \\
{ }^{440} \text { VDELCNA }\end{array}$ \\
\hline A0A0A0LFR2 & $\begin{array}{c}\text { Acetyltransferase component of pyruvate } \\
\text { dehydrogenase complex }\end{array}$ & $58 \mathrm{kDa}$ & $\begin{array}{c}\text { NRSQFLQC }{ }^{75} \text { QRGVSMM YYLTVDTC }^{341} \text { VDKLMDL } \\
\text { FMSVTLSC }^{509} \text { DHRVIDG }\end{array}$ \\
\hline A0A0A0KHD6 & Aconitate hydratase & $95 \mathrm{kDa}$ & PAVVDLAC ${ }^{103}$ MRDAMNR ALVAKKAC ${ }^{442}$ ELGLEVK \\
\hline $\mathrm{A} 0 \mathrm{~A} 0 \mathrm{~A} 0 \mathrm{KJ} 21$ & Actin & $41 \mathrm{kDa}$ & EDIQPLVC ${ }^{12}$ DNGTGMV TYNSIMKC ${ }^{287}$ DVDIRKD \\
\hline A0A0A0KRC5 & Acyl-coenzyme A oxidase & $73 \mathrm{kDa}$ & $\begin{array}{c}\text { QHLMESTC }^{457}{ }^{\text {KVQKAED SARMSVEC }}{ }^{486} \text { AKRLSQF } \\
\text { KDQLQKLC }{ }^{544} \text { SIYALFT }\end{array}$ \\
\hline A0A0A0LNE3 & Adenosylhomocysteinase & $53 \mathrm{kDa}$ & EMPGLMAC ${ }^{42}$ RTEFGPS \\
\hline A0A0A0KSC6 & Adenylosuccinate lyase & $60 \mathrm{kDa}$ & MEIGANC ${ }^{7}$ RVLDQPR LEFFHFSC ${ }^{186}$ TSEDINN \\
\hline A0A0A0K9F9 & Aldehyde dehydrogenase family 7 member B4 & $54 \mathrm{kDa}$ & QYMRRSTC ${ }^{490}$ TINYGNE \\
\hline H6WX41 & Alkaline alpha galactosidase 3 & $86 \mathrm{kDa}$ & HHTDAVYC ${ }^{441}$ AKQTAVV SSAKPRQC ${ }^{744}$ IVDSSVV \\
\hline A0A0A0КMH9 & Alpha-mannosidase & $114 \mathrm{kDa}$ & $\begin{array}{c}\text { MEKQANSC }{ }^{8} \text { LPFSFLV NNSIQGAC }^{76} \text { VQNVLDS }^{\text {QPKILSQC }}{ }^{470} \text { PLLNISF }\end{array}$ \\
\hline A0A0A0L5C9 & Aminopeptidase & $99 \mathrm{kDa}$ & QPSSIQAC ${ }^{82}$ EVSQILV AFALSMAC ${ }^{587}$ QQSVTSL \\
\hline A0A0A0KF04 & Aminotransferase 2 & $44 \mathrm{kDa}$ & DHTIKAVC ${ }^{142}$ IVHNETA \\
\hline A0A0A0LEK8 & Aspartate aminotransferase & $50 \mathrm{kDa}$ & NRVTTVQC ${ }^{163}$ LSGTGSL \\
\hline G3EIZ8 & ATP synthase subunit alpha & $54 \mathrm{kDa}$ & AESETLYC ${ }^{202}$ VYVAIGQ \\
\hline A0A2D0UXD2 & Betaine aldehyde dehydrogenase & $54 \mathrm{kDa}$ & AKLEAIDC ${ }^{100}$ GKPLEEA \\
\hline $\mathrm{A} 0 \mathrm{~A} 0 \mathrm{~A} 0 \mathrm{~K} 2 \mathrm{H} 5$ & Beta-xylosidase/alpha-L-arabinofuranosidase 2-like & $84 \mathrm{kDa}$ & LAGLDLDC $^{344}$ GDFLGKH PGCANVAC ${ }^{485}$ TSAQLDE \\
\hline A0A0A0KYI1 & Biotin carboxylase & $58 \mathrm{kDa}$ & $\begin{array}{c}\text { MDAAMPLC }{ }^{8} \text { KSARAPS KLADESVC }^{117} \text { IGEAPSS } \\
\text { SAAVSRGC }{ }^{142} \text { TMLHPGY }\end{array}$ \\
\hline A0A0A0LD02 & Carbonic anhydrase & $35 \mathrm{kDa}$ & STASINTC ${ }^{9}$ LFSLNKS ACSDSRVC ${ }^{167}$ PSHVLDF \\
\hline G8EX76 & Chloroplast transketolase & $80 \mathrm{kDa}$ & EGIANEAC ${ }^{246}$ SLAGHWG \\
\hline
\end{tabular}


Table 1. Cont

\begin{tabular}{|c|c|c|c|}
\hline Accession Number & Protein Name & Mol Mass & Peptide Sequence \\
\hline A0A0A0LCU8 & Coatomer subunit beta & $106 \mathrm{kDa}$ & $\begin{array}{c}\text { MEKSC }^{5} \text { TLLVHFD STAVIYEC } \\
\text { R62 AGTLVSL } \\
\text { RAAANTYC }{ }^{284} \text { QLLLSQS MKSTNMKC }^{879} \text { LTPISAL }\end{array}$ \\
\hline A0A0A0LBW6 & D-3-phosphoglycerate dehydrogenase & $63 \mathrm{kDa}$ & AAATEHGC ${ }^{144}$ LVVNAPT \\
\hline A0A0A0KG56 & Dihydrolipoyl dehydrogenase 2, chloroplastic-like & $59 \mathrm{kDa}$ & KLVPHVYC ${ }^{393}$ IGDANGK \\
\hline A0A0A0LTJ3 & Elongation factor Ts, mitochondrial & $121 \mathrm{kDa}$ & TGAGMMDC ${ }^{693}$ KKALAES TGAGMMDC ${ }^{936}$ KKALSET \\
\hline A0A0A0K581 & Eukaryotic translation initiation factor 3 subunit B & $60 \mathrm{kDa}$ & TTKTLGYC ${ }^{112}$ FIEYGTP \\
\hline A0A0A0LC36 & Eukaryotic translation initiation factor 3 subunit $C$ & $106 \mathrm{kDa}$ & TKARAMLC ${ }^{519}$ DIYHHAL SWDQPSGC ${ }^{785}$ IIFHDVT \\
\hline A0A0A0L3P3 & Ferredoxin-NADP reductase, chloroplastic & $46 \mathrm{kDa}$ & DSKTVSLC ${ }^{213}$ VKRLVYT \\
\hline $\mathrm{A} 0 \mathrm{~A} 0 \mathrm{~A} 0 \mathrm{~K} 8 \mathrm{H} 3$ & Fructose-1,6-bisphosphatase, cytosolic & $36 \mathrm{kDa}$ & LVSSGRTC ${ }^{95}$ ILVSEED \\
\hline A0A0A0KKE4 & Fructose-bisphosphate aldolase & $38 \mathrm{kDa}$ & MSC ${ }^{3}$ YRGKYAD \\
\hline A0A0A0KEY8 & Glucose-1-phosphate adenylyltransferase & $57 \mathrm{kDa}$ & PNLKRKLC ${ }^{58}$ ISSLIAD \\
\hline A0A0A0LRW2 & Glucose-6-phosphate isomerase & $67 \mathrm{kDa}$ & MASISGIC ${ }^{8}$ SSSPSLK AVLNEASC ${ }^{559}$ KEPVEPL \\
\hline A0A0A0KPY1 & Glutamate decarboxylase & $56 \mathrm{kDa}$ & MVDENTIC ${ }^{205}$ VAAILGS KKKTNGVC ${ }^{499}$ \\
\hline A0A0A0K 488 & $\begin{array}{c}\text { Glutamate-1-semialdehyde 2,1-aminomutase 2, } \\
\text { chloroplastic-like }\end{array}$ & $54 \mathrm{kDa}$ & SVGIGLPC ${ }^{47}$ STKLSHT \\
\hline A0A0A0K8Q7 & Glutathione reductase & $59 \mathrm{kDa}$ & AGGVGGTC ${ }^{122}$ VIRGCVP \\
\hline $\mathrm{A} 0 \mathrm{~A} 0 \mathrm{~A} 0 \mathrm{~K} 8 \mathrm{C} 1$ & Glyceraldehyde-3-phosphate dehydrogenase & $36 \mathrm{kDa}$ & NIVSNASC ${ }^{154}$ TTNCLAP NASCTTNC ${ }^{158}$ LAPLAKV \\
\hline A0A0A0LN17 & Glycine cleavage system $P$ protein & $113 \mathrm{kDa}$ & TFVISNNC ${ }^{252}$ HPQTIDI NPASAAMC ${ }^{688}$ GMKIVSV \\
\hline A0A0A0LAN5 & Glycosyltransferase & $55 \mathrm{kDa}$ & QLTPRPNC ${ }^{123}$ IISDMCI \\
\hline A0A0A0KHX5 & $\begin{array}{c}\text { Glyoxysomal fatty acid beta-oxidation multifunctional } \\
\text { protein MFP-a }\end{array}$ & $79 \mathrm{kDa}$ & MC $^{2}$ HALLVTI NLKHTIAC ${ }^{303}$ IDAVETG \\
\hline A0A0A0LNA7 & $\begin{array}{c}\text { Guanosine nucleotide diphosphate dissociation } \\
\text { inhibitor }\end{array}$ & $49 \mathrm{kDa}$ & SEGETAKC ${ }^{278}$ KKVVCDP \\
\hline A0A0A0K921 & Heat shock $70 \mathrm{kDaa}$ protein 15 -like & $92 \mathrm{kDa}$ & VIDQLVYC ${ }^{704}$ INSYREA \\
\hline A0A0A0KXG3 & Heat shock protein 70 & $70 \mathrm{kDa}$ & $\begin{array}{c}\text { NMDLFRKC }{ }^{319} \text { MEPVEKC CMEPVEKC }{ }^{326} \text { LRDAKMD } \\
\text { MKELESIC }{ }^{609} \text { NPIIAKM }\end{array}$ \\
\hline A0A0A0K5T7 & Ketol-acid reductoisomerase & $63 \mathrm{kDa}$ & NISVIAVC ${ }^{242}$ PKGMGPS CMDILYEC ${ }^{394}$ YEDVASG \\
\hline A0A0A0LXB9 & L-ascorbate oxidase & $65 \mathrm{kDa}$ & $\begin{array}{l}\text { YMFWSPDC }{ }^{54} \text { VENIVMG GTASISQC }{ }^{116} \text { AINPGET } \\
\text { ELSGKEKC }{ }^{236} \text { APFILHV IPPKALAC }{ }^{574} \text { GSTALVK }\end{array}$ \\
\hline A0A0A0L5B9 & Lon protease homolog 2, peroxisomal & $98 \mathrm{kDa}$ & DLKLASAC ${ }^{757}$ ESNLLEG \\
\hline A0A0A0LR30 & Lsocitrate lyase & $64 \mathrm{kDa}$ & QLKTFSEC ${ }^{320}$ VTDAIMN \\
\hline A0A0A0L0E4 & Malate dehydrogenase & $36 \mathrm{kDa}$ & CTAIAKYC ${ }^{142}$ PNALVNM \\
\hline A0A0A0LUC5 & Malate synthase & $65 \mathrm{kDa}$ & KGMYKEAC ${ }^{533}$ KMFTRQC \\
\hline
\end{tabular}


Table 1. Cont

\begin{tabular}{|c|c|c|c|}
\hline Accession Number & Protein Name & Mol Mass & Peptide Sequence \\
\hline A0A0A0L5H2 & Methionine S-methyltransferase & $120 \mathrm{kDa}$ & 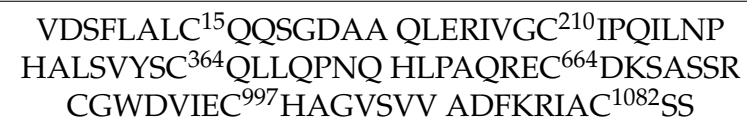 \\
\hline A0A0A0LEZ3 & Methionine synthase & $84 \mathrm{kDa}$ & 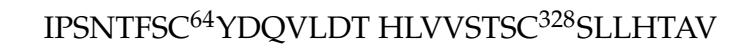 \\
\hline A0A0A0LIC6 & Methylenetetrahydrofolate reductase & $72 \mathrm{kDa}$ & ETMMHLTC $^{128}$ TNMPVEK YEKFMKYC $^{446}$ LGKLRSS \\
\hline A0A0A0KI79 & Mg-protoporphyrin IX chelatase & $45 \mathrm{kDa}$ & KGRPQVQC ${ }^{60}$ NVATEIN KVKISRVC ${ }^{350}$ AELNVDG \\
\hline A0A0A0LN97 & Multicopper oxidase & $60 \mathrm{kDa}$ & DGVYGTTC ${ }^{99}$ PIPPGKN \\
\hline A0A0A0KIJ0 & Ncharacterized protein & $55 \mathrm{kDa}$ & IEPVPESC ${ }^{99}$ VSTLEER \\
\hline A0A0A0L679 & Phospho-2-dehydro-3-deoxyheptonate aldolase & $57 \mathrm{kDa}$ & FLLQGGDC ${ }^{124}$ AESFKEF NSRYHTHC ${ }^{479}$ DPRLNAS \\
\hline A0A0A0KEF3 & Phosphoglycerate kinase & $50 \mathrm{kDa}$ & QVVKADDC ${ }^{177}$ IGPEVEK \\
\hline A0A0A0KTJ4 & Phospholipase D & $92 \mathrm{kDa}$ & $\begin{array}{c}\text { YFSQRRGC }{ }^{178} \text { KVTLYQD KFYEPHRC }^{209} \text { WEDVFDA } \\
\text { LFPESIEC }^{736} \text { VKSVNQL }\end{array}$ \\
\hline A0A0A0L987 & Phosphoribulokinase & $46 \mathrm{kDa}$ & $* * * *$ MAVC $^{4}$ TVYTTQS \\
\hline A0A0A0L989 & Polyadenylate-binding protein & $71 \mathrm{kDa}$ & AFGSILSC ${ }^{146}$ KVALDSS \\
\hline A0A0A0K809 & $\begin{array}{l}\text { Presequence protease } 1, \\
\text { chloroplastic/mitochondrial-like }\end{array}$ & $122 \mathrm{kDa}$ & $\begin{array}{c}\text { VFLRSLTC }^{12} \text { SSLVCNR RGKAMSGC }^{743} \text { AEDLFNL } \\
\text { SLLSRKNC }{ }^{847} \text { LVNITAD }^{-1 T A}\end{array}$ \\
\hline A0A0A0K8X9 & Protease Do-like 2, chloroplastic & $68 \mathrm{kDa}$ & $\begin{array}{l}\text { AAAMASSC }{ }^{9} \text { FSPFDST VLARGVDC }{ }^{204} \text { DIALLSV } \\
\text { LKFGNLPC }{ }^{230} \text { LQDAVTV AAIAASSC }{ }^{571} \text { ILRDYGI }\end{array}$ \\
\hline A0A0A0LRK5 & Purple acid phosphatase & $54 \mathrm{kDa}$ & VLCDLGVC ${ }^{26}$ NGGITSG \\
\hline A0A0A0LOU0 & $\begin{array}{l}\text { Pyrophosphate-fructose 6-phosphate } \\
\text { 1-phosphotransferase subunit alpha }\end{array}$ & $67 \mathrm{kDa}$ & $\begin{array}{c}\text { ETFAEAKC }{ }^{208} \text { PTKVVGV ASHVALEC }{ }^{276} \text { TLQSHPN } \\
\text { RTIVKPGC }{ }^{584} \text { SQEVLKA }\end{array}$ \\
\hline A0A0A0КH95 & $\begin{array}{l}\text { Pyrophosphate-fructose 6-phosphate } \\
\text { 1-phosphotransferase subunit beta }\end{array}$ & $61 \mathrm{kDa}$ & LKTRVIGC ${ }^{224}$ PKTIDGD SFGFDTAC ${ }^{247}$ RIYAEMI \\
\hline A0A218KBQ1 & Pyruvate kinase & $55 \mathrm{kDa}$ & $\begin{array}{c}\text { KPGNNILC }^{143} \text { SDGTITL QKMMIYKC }{ }^{287} \text { NLAGKPV } \\
\text { AVLDGTDC }{ }^{328} \text { VMLSGES }\end{array}$ \\
\hline A0A0A0KAU8 & RuBisCO large subunit-binding protein subunit alpha & $64 \mathrm{kDa}$ & LSSASILC ${ }^{14}$ SSHKSLR \\
\hline A0A0A0KFZ8 & RuvB-like helicase & $51 \mathrm{kDa}$ & PQTKFVQC ${ }^{224}$ PDGELQK \\
\hline A0A0A0KBZ1 & S-(hydroxymethyl)glutathione dehydrogenase & $40 \mathrm{kDa}$ & TQGQVITC ${ }^{10}$ KAAVAWE GVDYSFEC ${ }^{271}$ IGNVNVM \\
\hline C4PAW8 & Sedoheptulose-1,7-bisphosphatase & $42 \mathrm{kDa}$ & GLIRLLTC ${ }^{93}$ MGEALRT SHFCKYAC ${ }^{148}$ SEEVPEL \\
\hline A0A0A0K8A3 & Selenium-binding protein 2-like & $53 \mathrm{kDa}$ & KDTGYVGC ${ }^{277}$ ALTSNMV \\
\hline A8CM21 & Stachyose synthase & $96 \mathrm{kDa}$ & SSAINKGC ${ }^{383}$ TSCSCKA GLTNMFNC $^{792}$ SGTIQHL \\
\hline A0A0A0KGA1 & Succinate-semialdehyde dehydrogenase & $58 \mathrm{kDa}$ & GPALASGC $^{230}$ TVVIKPS NSGQTC ${ }^{346}$ VCANRILVQ \\
\hline A0A0A0LVU2 & T-complex protein 1 subunit delta & $57 \mathrm{kDa}$ & RSLHDALC ${ }^{404}$ VVRCLVN AITLATEC $^{519}$ VRMILKI \\
\hline
\end{tabular}


Table 1. Cont

\begin{tabular}{|c|c|c|c|}
\hline Accession Number & Protein Name & Mol Mass & Peptide Sequence \\
\hline A0A0A0LZU0 & T-complex protein 1 subunit eta & $60 \mathrm{kDa}$ & FADRDIFC ${ }^{313}$ AGRVAEE NAATEAAC ${ }^{517}$ LILSVDE \\
\hline A0A0A0LLK5 & Tocopherol cyclase & $57 \mathrm{kDa}$ & PLCGIHHC ${ }^{16}$ SFKLVEA \\
\hline A0A0A0KBL8 & Transketolase, chloroplastic & $80 \mathrm{kDa}$ & NRSSRSRC ${ }^{65}$ GVVRASV EGIANEAC ${ }^{249}$ SLAGHWG \\
\hline A0A0A0K6A8 & Tubulin alpha chain & $49 \mathrm{kDa}$ & $\begin{array}{c}\text { GIQVGNAC }{ }^{20} \text { WELYCLE TIQFVDWC }{ }^{347} \text { PTGFKCG } \\
\text { AKVQRAVC }{ }^{376} \text { MISNSTS }\end{array}$ \\
\hline A0A0A0LCY8 & Tubulin beta chain & $50 \mathrm{kDa}$ & $\begin{array}{c}\text { LHIQGGQC }{ }^{12} \text { GNQIGAK ATMSGVTC }{ }^{238} \text { CLRFPGQ } \\
\text { NNVKSTVC }{ }^{354} \text { DIPPTGL }\end{array}$ \\
\hline A0A0A0K9N4 & Ubiquitin carboxyl-terminal hydrolase 6 & $54 \mathrm{kDa}$ & $\begin{array}{c}\text { YMNSTLQC }{ }^{121} \text { LHSVPEL MQQDAEEC }^{200} \text { WTQLLYT } \\
\text { ESVYSLKC }{ }^{256} \text { HISQEVN }\end{array}$ \\
\hline A0A0A0KZ30 & UDP-glucose 6-dehydrogenase & $52 \mathrm{kDa}$ & MVKIC ${ }^{5}$ CIGAGYV TKEAHAVC ${ }^{417}$ ILTEWDE \\
\hline A0A0A0KZU3 & Gamma aminobutyrate transaminase 2 & $56 \mathrm{kDa}$ & TNPKLGSC ${ }^{18}$ AKDVAAL \\
\hline AOAOAOLHRO & PALP domain-containing protein & $58 \mathrm{kDa}$ & SSPFTLVC ${ }^{36}$ SSATSDS \\
\hline A0A0A0LQL1 & Uncharacterized protein & $110 \mathrm{kDa}$ & LARGQLRC $^{391}$ IGATTLE \\
\hline A0A0A0LTW3 & UVR domain-containing protein & $96 \mathrm{kDa}$ & RRRKASRC ${ }^{26}$ VPRAMFE LARGELQC ${ }^{343}$ IGATTLD \\
\hline A0A0A0KSQ4 & Probable nucleoredoxin 1 & $63 \mathrm{kDa}$ & WICEGGVC 559 RKA \\
\hline A0A0A0L5E7 & Uncharacterized protein & $43 \mathrm{kDa}$ & QQFTGLRC ${ }^{13}$ APLSSSR \\
\hline A0A0A0LNR8 & Peptidase_S9 domain-containing protein & $85 \mathrm{kDa}$ & $\begin{array}{c}\text { ILSGEVSC }^{428} \text { ISPANSN PVKDVSNC }{ }^{514} \text { LTKGASE } \\
\text { AAARNPVC }{ }^{653} \text { NLALMVG }\end{array}$ \\
\hline A0A0A0KN12 & Oxalate-CoA ligase-like & $55 \mathrm{kDa}$ & KLRFIRS ${ }^{291}$ CSASLAPS \\
\hline A0A0A0K983 & Uncharacterized protein & $69 \mathrm{kDa}$ & TTDGKTNC $^{422}$ LNAAVGT AMVTQAYC ${ }^{569}$ DVPFSYT \\
\hline A0A0A0KI31 & $\begin{array}{l}\text { Glyoxysomal fatty acid beta-oxidation Multifunctional } \\
\text { protein MFP-a }\end{array}$ & $79 \mathrm{kDa}$ & GLEVAMAC ${ }^{124}$ HARLSTK NLKHPLVC ${ }^{251}$ IDVVETG \\
\hline A0A0A0KIK3 & enolase isoform & $47 \mathrm{kDa}$ & QIKTGAPC ${ }^{408}$ RSERLAK \\
\hline A0A0A0KL58 & AA_TRNA_LIGASE_II domain-containing protein & $51 \mathrm{kDa}$ & TATERTLC ${ }^{402}$ CILENYQ ATERTLC ${ }^{403}$ CILENYQK \\
\hline А0А0АОКРТ0 & Protein kinase domain-containing protein & $127 \mathrm{kDa}$ & RGAAKGLC ${ }^{974}$ FLHHNCI YLEITLRC ${ }^{1124}$ VEEFPSK \\
\hline A0A0A0KQJ3 & Alpha-amylase 3, chloroplastic isoform & $101 \mathrm{kDa}$ & $\begin{array}{l}\text { LDPLLYHC }{ }^{13} \text { AKGKHRF RPCSFTYC }{ }^{37} \text { PNKLLCH } \\
\text { NWELTVGC }{ }^{112} \text { NLAGKWI ISVSVRKC }{ }^{292} \text { SETTKYL }\end{array}$ \\
\hline A0A0A0КТН8 & Malate dehydrogenase, chloroplastic & $48 \mathrm{kDa}$ & $\begin{array}{c}\text { SRTSRVTC }{ }^{49} \text { SINQVEA CNTNALIC }{ }^{230} \text { LKNAPKI } \\
\text { ELLAEKRC }{ }^{413} \text { VAHLTGE }\end{array}$ \\
\hline A0A0A0KW04 & 2-hydroxyacyl-CoA lyase & $60 \mathrm{kDa}$ & DISEIPNC ${ }^{154}$ VARVLNS RSLAIGKC ${ }^{274}$ DVALVVG \\
\hline A0A0A0KWS0 & $\begin{array}{l}\text { 2,3-bisphosphoglycerate-independent } \\
\text { Phosphoglycerate mutase }\end{array}$ & $61 \mathrm{kDa}$ & NGVRTFAC ${ }^{356}$ SETVKFG \\
\hline A0A0A0L7Y5 & $11 S$ globulin seed storage protein 2-like & $57 \mathrm{kDa}$ & SSGLIVKC ${ }^{260}$ DEEMSFL NGIEETVC ${ }^{297}$ TARVQHN \\
\hline A0A0A0LFS9 & Cell division control protein 48 homolog $\mathrm{E}$ & $89 \mathrm{kDa}$ & CTEAALQC $^{426}$ IREKMDV KARQSAPC ${ }^{576}$ VLFFDEL $^{2}$ \\
\hline A0A0A0LHX3 & Uncharacterized protein & $71 \mathrm{kDa}$ & NTPQQLAC ${ }^{176}$ IDVIEDG KVPLCIPC ${ }^{201}$ EDKVFRE \\
\hline
\end{tabular}


Table 1. Cont

\begin{tabular}{|c|c|c|c|}
\hline Accession Number & Protein Name & Mol Mass & Peptide Sequence \\
\hline A0A0A0LJ13 & Triosephosphate isomerase, chloroplastic & $32 \mathrm{kDa}$ & EGLGVIAC ${ }^{177}$ IGELLEE \\
\hline A0A0A0LTR4 & Beta-glucosidase 44 -like & $57 \mathrm{kDa}$ & LPVVCMLC ${ }^{14}$ AATAMHL \\
\hline A0A0A0LV53 & Lysosomal beta glucosidase-like & $68 \mathrm{kDa}$ & NVCSNVNC ${ }^{542}$ VVVVVSG \\
\hline A0A0A0KD01 & Uncharacterized protein & $91 \mathrm{kDa}$ & $\begin{array}{c}\text { HLNAAASC }{ }^{154} \text { QIQFVCK KELDEAIC }{ }^{328} \text { WAKVSET } \\
\text { NLEDRLAC }{ }^{546} \text { KDNSSPL }\end{array}$ \\
\hline A0A0A0KTK6 & Aminotran_1_2 domain-containing protein & $52 \mathrm{kDa}$ & KVPDVLYC ${ }^{417}$ LKLLEAT \\
\hline A0A0A0KW15 & Uncharacterized protein & $51 \mathrm{kDa}$ & EIKEGCGC ${ }^{460} \mathrm{KG}$ \\
\hline A0A0A0L0K6 & Uncharacterized protein & $50 \mathrm{kDa}$ & DGVYGTTC ${ }^{103}$ PIPPGKN \\
\hline A0A0A0L0Q4 & Ribos_L4_asso_C domain-containing protein & $44 \mathrm{kDa}$ & QGAFGNMC ${ }^{100}$ RGGRMFA \\
\hline A0A0A0L5U9 & Acyl-CoA dehydrogenase family member 10 & $91 \mathrm{kDa}$ & $\begin{array}{l}\text { STVGNQMC } 262 \text { DVAYFCL NLEYGHLC }{ }^{511} \text { EIMGRSI } \\
\text { SDATNIEC }^{579} \text { SITREGD SGAMDPRC }{ }^{605} \text { KILIVMG }\end{array}$ \\
\hline A0A0A0LI90 & Aldedh domain-containing protein & $53 \mathrm{kDa}$ & HKAPIAEC ${ }^{98}$ LVKEIAK \\
\hline A0A0A0LRM4 & 11-beta-hydroxysteroid dehydrogenase 1B-like & $38 \mathrm{kDa}$ & PVETADEC ${ }^{267}$ AKGVVRG \\
\hline A0A0A0LUA8 & Aldedh domain-containing protein & $59 \mathrm{kDa}$ & KVGPALAC ${ }^{232}$ GNTVVLK GKSPFIVC $^{325}$ EDADVDK \\
\hline A0A0A0K8W3 & Uncharacterized protein & $109 \mathrm{kDa}$ & MKNC ${ }^{4}$ SNALSAN KLLRNYRC ${ }^{701}$ HPDILHL \\
\hline A0A0A0KNB1 & OMPdecase domain-containing protein & $52 \mathrm{kDa}$ & STSYDLVC ${ }^{73}$ GVPYTAL EKIGPEIC $^{274}{ }^{2 L K T H V D ~}$ \\
\hline A0A0A0L1I8 & DNA mismatch repair protein MLH3 isoform & $136 \mathrm{kDa}$ & $\begin{array}{c}\text { AYVLNLEC }^{311} \text { PVSFYDL KKSRMQSC }^{394} \text { QASLIDS } \\
\text { RVLNSKAC }{ }^{1128} \text { RGAIMFG }\end{array}$ \\
\hline A0A0A0LAP3 & Uncharacterized protein & $60 \mathrm{kDa}$ & MVTHC ${ }^{5}$ INLHLHR \\
\hline A0A0A0LL68 & E2F_TDP domain-containing protein & $47 \mathrm{kDa}$ & ALALPPQC ${ }^{47}$ CLQYHRP ACFSERQC ${ }^{318}$ RMIIKST \\
\hline A0A0A0LPD2 & B5 domain-containing protein & $66 \mathrm{kDa}$ & ANRYDLLC ${ }^{76}$ LEGLAQA TKNVFIEC ${ }^{256}$ TATDLTK \\
\hline A0A0A0LRD9 & Programmed cell death protein 4 & $78 \mathrm{kDa}$ & DTFEACRC ${ }^{309}$ IRQLGVT VVSEACQC ${ }^{606}$ IRDLGMP \\
\hline A0A0A0LSH7 & DEAD-box ATP-dependent RNA helicase 56 & $48 \mathrm{kDa}$ & KDLLKNEC ${ }^{166}$ PHIVVGT \\
\hline A0A0A0LYN5 & Asparagine-tRNA ligase, cytoplasmic 1 & $64 \mathrm{kDa}$ & LQVETYAC ${ }^{324}$ ALSSVYT DLQDDMNC ${ }^{368}$ AEAYVRF \\
\hline A0A0A0LYR4 & Arginine-tRNA ligase, cytoplasmic isoform & $66 \mathrm{kDa}$ & AEVVEEAC ${ }^{526}$ TNLLPNV \\
\hline A0A0A0KMJ3 & Uncharacterized protein & $111 \mathrm{kDa}$ & $\begin{array}{c}\text { MARLVLPC }{ }^{8} \text { KSVGLAR QASRKLIC }^{80} \text { SVATEPL } \\
\text { DIMAKYTC }{ }^{241} \text { RIEADKS }\end{array}$ \\
\hline A0A0A0KSN9 & T-complex protein 1 subunit zeta 1 & $59 \mathrm{kDa}$ & MERLVLAC ${ }^{331}$ GGEAVNS NVKNPHSC ${ }^{375}$ TILIKGP \\
\hline A0A0A0L246 & Uncharacterized protein & $57 \mathrm{kDa}$ & $\begin{array}{l}\text { LEDTLVAC }{ }^{63} \text { LDRIFKT RSRAMVIC }{ }^{278} \text { GRLLSKE } \\
\text { FSLVDESC }{ }^{295} \text { LRNLISA LLSSFPTC }{ }^{345} \text { VKHVIYA }\end{array}$ \\
\hline A0A0A0L3I1 & Peptidase_S9 domain-containing protein & $81 \mathrm{kDa}$ & $\begin{array}{c}\text { MSPC }^{4} \text { ALLRLFR VKEGDEPC }{ }^{132} \text { DITPKEF } \\
\text { NFVDKFSC }{ }^{651} \text { PIILFQG }\end{array}$ \\
\hline A0A0A0L6P6 & HATPase_c domain-containing protein & $80 \mathrm{kDa}$ & $\begin{array}{c}\text { KKSFENLC }^{548} \text { KTIKDIL DRIVDSPC }^{573} \text { CLVTGEY } \\
\text { RIVDSPC }^{574} \text { CLVTGEYG }\end{array}$ \\
\hline
\end{tabular}


Table 1. Cont

\begin{tabular}{|c|c|c|c|}
\hline Accession Number & Protein Name & Mol Mass & Peptide Sequence \\
\hline A0A0A0LFM9 & T-complex protein 1 subunit theta & $58 \mathrm{kDa}$ & KYAADAVC ${ }^{516}$ TVLRVDQ \\
\hline A0A0A0LIF5 & Chaperonin CPN60-2, mitochondrial & $61 \mathrm{kDa}$ & VAGDGTTC $^{122}$ ATILTRA TNQKNQKC ${ }^{244}$ ELEDPLI \\
\hline A0A0A0LXZ3 & UVR domain-containing protein & $102 \mathrm{kDa}$ & LARGELQC ${ }^{404}$ IGATTLD \\
\hline A0A0A0M2C3 & $\begin{array}{c}\text { RuBisCO large subunit-binding protein subunit beta, } \\
\text { chloroplastic }\end{array}$ & $64 \mathrm{kDa}$ & MAVEYENC ${ }^{280}$ KLLLVDK KTFLMSDC $^{584}$ VVVEIKE \\
\hline A0A0A0KSV2 & $\begin{array}{c}\text { Bifunctional aspartokinase/homoserine dehydrogenase } \\
1 \text {, chloroplastic }\end{array}$ & $101 \mathrm{kDa}$ & QVAVIPNC ${ }^{490}$ SILAAVG \\
\hline A0A0A0KWR4 & Probable serine protease EDA2 isoform & $55 \mathrm{kDa}$ & MDLWLSEC ${ }^{480} \mathrm{QSTTGRN}$ \\
\hline A0A0A0LCI7 & $\begin{array}{l}\text { 5-methyltetrahydropteroyltriglutamate-Homocysteine } \\
\text { methyltransferase-like }\end{array}$ & $84 \mathrm{kDa}$ & HLVVSTSC ${ }^{328}$ SLLHTAV \\
\hline A0A0A0LK02 & SET domain-containing protein & $57 \mathrm{kDa}$ & RANEELIC ${ }^{413}$ QVVRNAC \\
\hline A0A0A0LZR2 & $\begin{array}{c}\text { 5-methyltetrahydropteroyltriglutamate-homocysteine } \\
\text { methyltransferase }\end{array}$ & $91 \mathrm{kDa}$ & KIVVSTSC ${ }^{390}$ SLLHTAV \\
\hline A0A0A0M063 & Glyco_transf_20 domain-containing protein & $97 \mathrm{kDa}$ & LVKELSEC ${ }^{861}$ SVSNLS \\
\hline $\mathrm{A} 0 \mathrm{~A} 0 \mathrm{~A} 0 \mathrm{KK} 36$ & Probable polygalacturonase & $48 \mathrm{kDa}$ & WNIHPVYC ${ }^{208}$ RNVVVRY \\
\hline A0A0A0LHX3 & $\begin{array}{l}\text { Peroxisomal fatty acid beta-oxidation multifunctional } \\
\text { protein AIM1 isoform }\end{array}$ & $71 \mathrm{kDa}$ & NTPQQLAC ${ }^{176}$ IDVIEDG KVPLCIPC ${ }^{201}$ EDKVFRE \\
\hline A0A0A0LL68 & legumin J & $47 \mathrm{kDa}$ & ALALPPQC ${ }^{47}$ CLQYHRP ACFSERQC ${ }^{318}$ RMIIKST \\
\hline A0A0A0LNN6 & Uncharacterized protein & $55 \mathrm{kDa}$ & NGFEETVC ${ }^{313}$ TLRLKHS \\
\hline A0A0A0L6K0 & Uncharacterized protein & $37 \mathrm{kDa}$ & $\begin{array}{c}\text { GFVFPKKC }^{75} \text { NEVVIKL PEYVQKSC }^{147} \text { SLNQEET } \\
\text { AGEEGLEC }{ }^{293} \text { ISMIVAT }\end{array}$ \\
\hline A0A0A0L7C4 & $\begin{array}{l}\text { Acetyl-coenzyme A synthetase, } \\
\text { chloroplastic/glyoxysomal isoform }\end{array}$ & $89 \mathrm{kDa}$ & $\begin{array}{l}\text { NLIVTSSC }^{10} \text { NAVRPFP SSTTTSSC }{ }^{75} \text { LLRPPFA } \\
\text { LAQRIIDC }^{329} \text { KPKIVIT LVSHPQC } \\
{ }^{699} \text { AEAAVVG }\end{array}$ \\
\hline A0A0A0LBK4 & 3-ketoacyl-CoA thiolase 2 , peroxisomal & $47 \mathrm{kDa}$ & LGTTGARC ${ }^{401}$ VATLLSE \\
\hline A0A0A0LT72 & NAB domain-containing protein & $40 \mathrm{kDa}$ & RTSSSPSC ${ }^{20}$ DTFSSNR KAGEMARC ${ }^{248}$ MLKLRDD \\
\hline A0A0A0LU46 & Probable aspartyl aminopeptidase & $56 \mathrm{kDa}$ & AATNDAKC ${ }^{36}$ KNNAVVT VVRNDMSC ${ }^{449}$ GSTIGPI \\
\hline A0A0A0LXJ8 & $\begin{array}{l}\text { 4-hydroxy-3-methylbut-2-en-1-yl Diphosphate synthase } \\
\text { (ferredoxin), chloroplastic }\end{array}$ & $82 \mathrm{kDa}$ & VALRVAEC ${ }^{181}$ FDKIRVN \\
\hline A0A0A0KGD1 & Elongation factor 2-like & 84 kDa & ETVEDVPC ${ }^{355}$ GNTVAMV \\
\hline A0A0A0L9F9 & WD_REPEATS_REGION domain-containing protein & $120 \mathrm{kDaa}$ & MAC ${ }^{3}$ IKGVNRS \\
\hline A0A0A0KTQ0 & PKS_ER domain-containing protein & 40 kDa & PSQLNSYC ${ }^{16} \mathrm{HFISSKL}$ \\
\hline $\mathrm{A} 0 \mathrm{~A} 0 \mathrm{~A} 0 \mathrm{KN} 12$ & Oxalate-CoA ligase-like & $55 \mathrm{kDa}$ & KLRFIRSC ${ }^{291}$ SASLAPS \\
\hline A0A0A0LXU2 & 4-coumarate-CoA ligase-like 7 & $59 \mathrm{kDa}$ & IHSPKILC $^{165}$ FNDLVNM GRELMEEC ${ }^{326}$ ANNIPSA \\
\hline A0A0A0KEW1 & Agglutinin domain-containing protein & $53 \mathrm{kDa}$ & ENESSWPC ${ }^{93}$ TLFNFIP LLATKAKC ${ }^{419}$ DIPFSYT \\
\hline
\end{tabular}


Table 1. Cont

\begin{tabular}{|c|c|c|c|}
\hline Accession Number & Protein Name & Mol Mass & Peptide Sequence \\
\hline А0А0A0КНT0 & F-box domain-containing protein & $45 \mathrm{kDa}$ & RLLLLRRC ${ }^{66}$ YSTATKK \\
\hline A0A0A0KLY1 & ANK_REP_REGION domain-containing protein & $56 \mathrm{kDa}$ & MC ${ }^{2}$ SGSKNKV KVDVNRAC ${ }^{109}$ GSDLTTA \\
\hline A0A0A0KT59 & Uncharacterized protein & $89 \mathrm{kDa}$ & $\begin{array}{l}\text { PCGLSLSC }^{66} \text { SLSLSLS DKAVESLC } \\
\text { AGKVTKFC }^{317} \text { RILSPSQL AIQHILPC } \\
\text { A }^{53} \text { VKELSSD }\end{array}$ \\
\hline A0A0A0KZ23 & PCI domain-containing protein & $37 \mathrm{kDa}$ & TRNYSEKC ${ }^{105}$ INNIMDF \\
\hline A0A0A0LQN5 & Minotran_1_2 domain-containing protein & $52 \mathrm{kDa}$ & PGNPTGQC ${ }^{226}$ LSEANLR \\
\hline A0A0A0LBA6 & Starch branching enzyme I & $99 \mathrm{kDa}$ & FPAVPPLC ${ }^{17}$ KRSDSTF \\
\hline A0A0A0KM90 & Uroporphyrinogen decarboxylase & $43 \mathrm{kDa}$ & MSC $^{3}$ IHNSPLP IHNSPLPC ${ }^{11}$ FSASSSS \\
\hline A0A0A0K6R4 & V-type proton ATPase catalytic subunit A & $68 \mathrm{kDa}$ & AIPGAFGC ${ }^{256}$ GKTVISQ \\
\hline
\end{tabular}


2.5. Effect of GSNO on the Activities and S-Nitrosylation Level of Tubulin Alpha Chain (TUA), Glutathione Reductase (GR), and Glyceraldehyde-3-Phosphate Dehydrogenase (GAPDH) During Adventitious Rooting

Here, tubulin alpha chain (TUA), glutathione reductase (GR), and glyceraldehyde-3-phosphate dehydrogenase (GAPDH) were selected as candidate proteins to further assess the level of nitrosylation during NO-induced adventitious rooting in cucumber. At 6 h, GSNO treatment significantly increased the expression level of TUA, and remarkably decreased the expression level and activities of GR and GAPDH (Figure 5A-E). However, exogenous application of GSNO significantly enhanced the nitrosylation level of these proteins, which was detected by the biotin switch technique (Figure 5D). On the contrary, the $S$-nitrosylation level of these proteins was largely blocked by the treatment of cPTIO (Figure 5D). Interestingly, removal of endogenous NO significantly inhibited the expression level of TUA but improved the expression level and activities of GR and GAPDH during the development of adventitious roots in cucumber (Figure 5A-E).
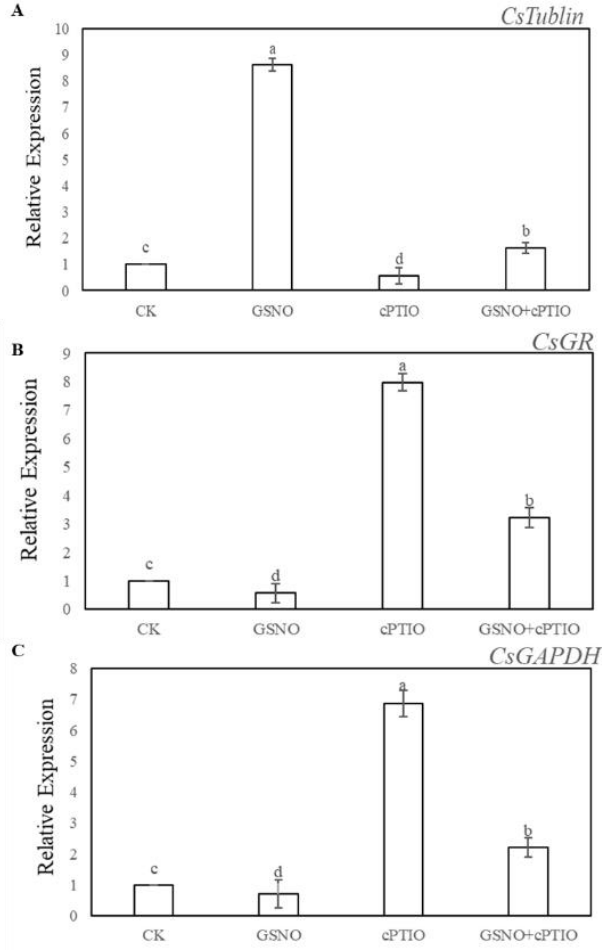

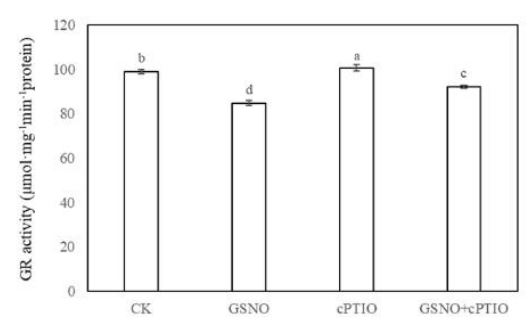

E
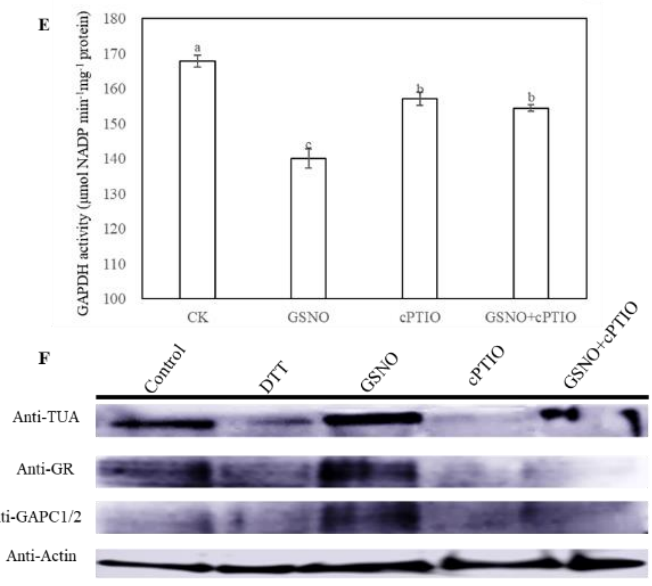

Figure 5. Effect of GSNO on the expression levels, enzymatic activities and S-nitrosylation level of tubulin alpha chain (TUA), glutathione reductase (GR), and glyceraldehyde-3-phosphate dehydrogenase (GAPDH) during adventitious rooting. Explants were incubated with distilled water (CK) or $200 \mu \mathrm{M}$ cPTIO, $50 \mu \mathrm{M}$ GSNO, or $50 \mu \mathrm{M}$ GSNO + $200 \mu \mathrm{M}$ cPTIO. TUA, GR and GAPDH expression level (A, B, C), and GR and GADPH activity (D, E) in cucumber explants was determined at $6 \mathrm{~h}$ of treatment. Immunoblot analysis of $S$-nitrosylated proteins in vivo (F). After biotinylation, proteins were purified with neutravidin-agarose, separated by sodium dodecyl sulfate-polyacrylamide gel (SDS-PAGE), and immunoblotted with anti-TUA, anti-GR, and anti-GAPDH antibodies. Bars with different lowercase letters were significantly different by Duncan's multiple range test $(p<0.05)$. Bars with different lowercase letters were significantly different by Duncan's multiple range test.

\section{Discussion}

In our study, the data presented herein demonstrated the evidence that there is a molecular mechanism of NO function to induce the development of adventitious rooting in cucumber. As previously reported in other researches, NO might play a critical role in affecting the root development [24-26]. For example, Yuan et al. [27] found the level of endogenous NO might be enhanced under cadmium (Cd) stress to inhibit the growth of root meristem in Arabidopsis through 
regulating auxin accumulation and transport. Alternatively, NO might act as a necessary factor affecting adventitious rooting [28-30]. According to our results, $\mathrm{NO}$ was indispensable for promoting adventitious rooting in cucumber (Figures 1 and 2). Interestingly, research suggested that NO could partly exert its influence on the process of root growth and development through S-nitrosylation [31-33]. To investigate potential NO regulation of physiological processes through modifying cysteine residues of proteins [19], the changes of S-nitrosylation level and endogenous NO content during adventitious rooting of cucumber explants were analyzed (Figure 3). Application of exogenous NO significantly increased the level of endogenous SNO and endogenous NO production. However, SNO and NO level with cPTIO treatment significantly were lower than those of the control and GSNO treatment, implying that $\mathrm{NO}$ might enhance the endogenous nitrosylation level during adventitious rooting (Figure 3A,C-E). Previously, Wang et al. [32] found that SNP could enhance the level of SNO. Our results indicated that $\mathrm{NO}$ might affect process of adventitious rooting through enhancing the endogenous nitrosylation. Moreover, it is known that GSNOR can regulate global levels of $S$-nitrosylation [34,35] Additionally, Lin et al. [36] found that S-nitrosoglutathione reductase (OsGSNOR) overexpression transgenic plants were detected with a lower SNO content indicating that GSNOR might play a vital role in SNO homeostasis. As mentioned above, NO might inhibit the activity of GSNOR1 preventing S-nitrosoglutathione scavenging [35]. As depicted in Figure 3B, a lower GSNOR activity was detected in GSNO treatment, which also suggested that GSNOR regulates the total level of SNOs during NO-induced adventitious rooting in cucumber.

For a deeper insight, $S$-nitrosylated proteins were identified during adventitious rooting of cucumber (Figure 4; Table 1). Among these proteins, a large amount of the $S$-nitrosylated proteins identified were closely related to carbon and energy metabolism, implying this process could be regulated by $S$-nitrosylation, during adventitious rooting of cucumber. Previous research suggested that carbohydrates and nitrogen compounds might provide nutrition and energy during adventitious root formation and development [37]. In our study, for example, pyruvate kinase, malate dehydrogenase, and malate synthase were involved in the tricarboxylic acid (TCA) cycle, which acts as an iconic process for carbohydrate metabolism [38]. However, the molecular mechanisms of these protein functions during the development of adventitious roots are still not established. Here, our results imply that these proteins may be $S$-nitrosylated during NO-induced adventitious rooting of cucumber. Moreover, cytoskeleton change might affect cell shape and translocate organelles which could enhance cell response to intracellular and extracellular signaling [39]. Potential candidates of $S$-nitrosylation during adventitious rooting in cucumber are also related to cytoskeleton structure including tubulin $\alpha$ and tubulin $\beta$ [40]. Tubulin $\alpha$ and tubulin $\beta$ have been demonstrated to be $S$-nitrosylated in mammals and plants $[40,41]$. These results indicate that the $S$-nitrosylation of tubulin variants could act as an important mediator in NO-promoted development of adventitious roots in cucumber. Additionally, another cluster of potential candidates for $S$-nitrosylation includes metabolic enzymes such as GAPDH, glucose-6-phosphate isomerase, fructose-bisphosphate aldolase, phosphoglycerate kinase, and so on (Table 1). Previous studies have reported that $\mathrm{H}_{2} \mathrm{O}_{2}$ treatment might affect fructose-1,6-biphosphate aldolase and 2-phosphoglycerate hydrolase undergoing S-glutathionylation [42]. Meanwhile, Lindermayr et al. [19] suggested that the glycolysis-related enzymes are sensitive to S-nitrosylation. Thus, these metabolism enzymes, which are identified as targets for $S$-nitrosylation, imply that $S$-nitrosylation of metabolic proteins could mediate adventitious root development.

In our study, there were $116 S$-nitrosylated proteins from both control and GSNO treatments (Figure 4C). These proteins participated in different processes of cellular metabolism, such as lipid metabolism, transcription and translation, hormone response, and signaling transduction (Figure 4D,E). As a consequence, these $S$-nitrosylated proteins with different functions might play a vital role in affecting the process of adventitious rooting. As previously reported in Wang et al. [32], NO could inhibit the growth of primary roots through S-nitrosylation of plastidial GAPDH. Our results indicated that NO could enhance the $S$-nitrosylation level of GAPDH, however, it was shown to decrease the expression level and activity of GAPDH during adventitious rooting (Figure 5C,E). In animals, some 
research has demonstrated that NO could inhibit GAPDH activity through S-nitrosylation [43,44]. Additionally, GAPDH activity was clearly inhibited by exogenous NO during NO-repression of the process of primary root growth [24]. These results might indicate that GAPDH is a key target for NO-specific PTM. Furthermore, we demonstrated evidence for the first time that GR and TUA could be over-nitrosylated under NO treatment during adventitious rooting (Figure 5). According to a previous study, GR had been shown to play an essential role for cell redox homeostasis [45]. Moreover, TUA has been found to play an essential role in cytoskeleton development [46]. The development of adventitious roots may be closely related with cell division and cell growth [47]. As depicted in Figure 5A, NO significantly increased the expression level of TUA, suggesting that the cell cycle process plays a vital role during adventitious root growth [48]. In addition, Begara-Morales [49] found that chloroplastic and cytosolic GR in peas are S-nitrosylated by GSNO, however, NO-based modification did not significantly affect this protein activity. In a previous study on mammal cells, an inhibitory effect on GR activity was shown after exposure to GSNO for a longer time [50]. According to our results, GSNO significantly decreased the expression level and activity of GR during adventitious rooting, implying that $S$-nitrosylation of GR induced by GSNO might inhibit protein activity and this change could be related to the development of adventitious roots in cucumber. Although some S-nitrosylation of proteins during adventitious rooting have been identified, whether the activities and functions of these identified proteins have been changed due to $S$-nitrosylation directly, needs to be further investigated. In the future, these results could provide valuable information for future investigations.

\section{Materials and Methods}

\subsection{Plant Materials}

Cucumber (Cucumis sativus 'BaiLv 1') seeds were supplied by the Gansu Academy of Agricultural Sciences, Lanzhou, China. The seeds were germinated in petri dishes on filter papers soaked with distilled water and maintained at $25 \pm 1{ }^{\circ} \mathrm{C}$ for 6 days with a $14 \mathrm{~h}$ photoperiod (photosynthetically active radiation $=200 \mu \mathrm{mol} \mathrm{s}^{-1} \mathrm{~m}^{-2}$ ). After removing the primary roots of 6-day-old seedlings, the cucumber explants were then maintained under the same conditions of temperature and photoperiod for another 5 days under different treatments as indicated below. These media were changed every day in order to keep the solution fresh. The number and length of adventitious roots per explant were counted and measured.

\subsection{Treatments of Explants}

Explants were placed in petri dishes containing distilled water (control) and different concentrations of S-nitrosoglutathione (GSNO, a donor of NO, Sigma, St Louis, MO, USA) as indicated in Figure 1 and kept at $25 \pm 1{ }^{\circ} \mathrm{C} 200 \mu \mathrm{M}$ 2-(4-carboxy-2-phenyl)-4, 4, 5, 5-tetramethylimidazoline-1-oxyl-3-oxide (c-PTIO; Sigma, St Louis, $\mathrm{MO}, \mathrm{USA}), 100 \mu \mathrm{M}$ sodium nitrate $\left(\mathrm{NaNO}_{3}\right.$, degradation product of $\mathrm{NO}$, Solarbio, Beijing, China) was added alone and with a suitable concentration of GSNO. The concentrations of $\mathrm{NO}$ scavenger and $\mathrm{NaNO}_{3}$ were based on the results of a preliminary experiment.

\subsection{Determination of Endogenous SNO Content, NO Production, and GSNOR Activity}

SNO content was determined as described by Feechan et al. [34] with minor modifications. Fresh cucumber explants were homogenized with extraction buffer ( $50 \mathrm{mM}$ Tris-HCl, pH 8.0), $150 \mathrm{mM}$ $\mathrm{NaCl}$, and $1 \mathrm{mM}$ protease inhibitor phenylmethanesulfonyl fluoride (PMSF) in an ice bath for $20 \mathrm{~min}$. The centrifugation was performed at $10,000 \mathrm{rpm}$ for $15 \mathrm{~min}$ at $4{ }^{\circ} \mathrm{C}$. The absorbance of the mixture reaction, which includes $1.5 \mathrm{~mL}$ of the supernatant, $1.5 \mathrm{~mL}$ of $0.1 \% \mathrm{~N}$-(1-naphthyl)-ethylenediamine, and $1 \%$ sulfanilamide, with and without adding $\mathrm{HgCl}_{2}$, was taken for $20 \mathrm{~min}$ in the dark. SNO content was recorded photometrically at $540 \mathrm{~nm}$ [34].

The level of endogenous NO in cucumber hypocotyls at $6 \mathrm{~h}$ was detected by NO fluorescent probe 4-amino-5-methylamino-2', $7^{\prime}$ - diaminofluoresceindiacetate (DAF-FM DA) [51]. The hypocotyls were 
loaded with $5 \mu \mathrm{M}$ DAF-FM DA for $30 \mathrm{~min}$ at $37^{\circ} \mathrm{C}$ in the dark. The samples were then washed three times with fresh buffer. DAF-FM DA fluorescence was visualized using a laser scanning confocal microscope (Leica TCS SL; Leica Microsystems, Wetzlar, Hessen, Germany). The excitation wavelength was $488 \mathrm{~nm}$ and the emission wavelength was $515 \mathrm{~nm}$.

NO content was measured according to the Greiss reagent method with some modifications [52]. A quantity of $0.2 \mathrm{~g}$ of explants was finely frozen in liquid nitrogen with the extract mixture $(4 \mathrm{~mL}$ of $50 \mathrm{mM}$ ice cold acetic acid buffer, containing $4 \%$ zinc diacetate). After that, centrifugation was performed at $10,000 \times g$ for $15 \mathrm{~min}$ at $4{ }^{\circ} \mathrm{C}$, and then the supernatants were collected. For each sample, $0.1 \mathrm{~g}$ of charcoal was added. After that, the supernatants were filtered and collected again, and then 1 $\mathrm{mL}$ of the mixture was pipetted into $1 \mathrm{~mL}$ of Greiss reagent. They were allowed to react for $30 \mathrm{~min}$ at room temperature. Then the absorbance was assayed at $540 \mathrm{~nm}$.

S-nitrosoglutathione reductase (GSNOR) activity was measured using the method of Durner et al. [53]. Samples were homogenized with $20 \mathrm{mM}$ Tris- $\mathrm{HCl}$ (pH 8.0, $0.2 \mathrm{mM}$ NADH, and $0.5 \mathrm{mM}$ EDTA) and centrifuged at $10,000 \mathrm{rpm}$ for $20 \mathrm{~min}$ at $4{ }^{\circ} \mathrm{C}$. The reaction was started by adding GSNO and the absorbance of the sample was measured at $340 \mathrm{~nm}$.

\subsection{Biotin-Switch Assay and Identification of Biotinylated Proteins}

Cucumber explants were ground in liquid nitrogen, extracted by HEN-2 Buffer (250 mM Hepes- $\mathrm{NaOH}, \mathrm{EDTA}$, neocuproine and proteinase inhibitor), followed by centrifugation at $13,000 \mathrm{~g}$ for $10 \mathrm{~min}$ at $4{ }^{\circ} \mathrm{C}$. Then, extracted protein was incubated in blocking buffer $(250 \mathrm{mM}$ Hepes, EDTA, SDS, methylmethane thiosulphonate (MMTS)) for $30 \mathrm{~min}$ at $50{ }^{\circ} \mathrm{C}$ under dark conditions. Subsequently, the MMTS was removed by cold acetone. The protein was resuspended with HEN-1 buffer $(250 \mathrm{mM}$ Hepes, EDTA, SDS) and $1 \mathrm{mM}$ sodium ascorbate and biotin-HPDP (Sigma, St Louis, MO, USA) were added for labeling. The S-nitrosylated proteins were identified by LC-MS/MS and measured by immunoblot analysis [54].

\subsection{Western Blotting}

For western blot analysis, proteins from different treatments were resolved using SDS-PAGE on $12 \%$ polyacrylamide gels, and transferred to polyvinylidene difluoride membranes (PVDF, Novex, San Diego, CA, USA) utilizing a wet transfer device (BioRad, Barcelona, Spain) at $105 \mathrm{~V}$ for $70 \mathrm{~min}$ at $4{ }^{\circ} \mathrm{C}$. The immunoreaction was performed with rabbit polyclonal antibodies against Biotin (1:2500) (Agrisera, Vännäs, Sweden), TUA (1:5000) (Agrisera, Vännäs, Sweden), GR (1:5000) (Agrisera, Vännäs, Sweden), and GAPDH (1:2000) (Agrisera, Vännäs, Sweden), Actin (1:2500) (Agrisera, Vännäs, Sweden). The blot was incubated in secondary antibody (goat anti-rabbit IgG), diluted to 1:10,000, for $1 \mathrm{~h}$ at 25 ${ }^{\circ} \mathrm{C}$.

\subsection{GR, GAPDH Activity}

GR activity was determined according to Foyer et al. [55]. A $0.2 \mathrm{~g}$ quantity of explant was ground in liquid nitrogen with the extract mixture, followed by centrifugation at $12,000 \mathrm{~g}$ for $20 \mathrm{~min}$ at $4{ }^{\circ} \mathrm{C}$. Then, a total of $100 \mu \mathrm{L}$ of enzyme extract was transferred into $2 \mathrm{~mL}$ of reaction mixture ( $25 \mathrm{mM}$ sodium phosphate buffer, pH 7.0, 0.1 mM EDTA, $0.5 \mathrm{mM}$ oxidized glutathione (GSSG), $0.12 \mathrm{mM}$ NADPH). GR activity was evaluated by measuring the decrease in absorbance at $340 \mathrm{~nm}$ due to NADPH oxidation.

The measurement of GAPDH activity was according to the method of Piattoni et al. [56]. Crude protein extraction was performed with $200 \mu \mathrm{L}$ reaction buffer $(50 \mathrm{mM}$ Tris- $\mathrm{HCl}, \mathrm{pH} 8.5,10 \mathrm{mM}$ sodium arsenate, $2 \mathrm{mM} \mathrm{NAD}^{+}, 1 \mathrm{U} / \mathrm{mL}$ aldolase, $1.2 \mathrm{mM}$ fructose-1,6-diphosphate) at $30^{\circ} \mathrm{C}$. Then, the reaction was monitored at $340 \mathrm{~nm}$.

\subsection{Gene Expression Analyses by RT-qPCR}

The method of real time RT-PCT (RT-qPCR) analyses and statistical data analyses reference the procedure of Zhao et al. [57]. The cDNA was amplified using the following primers: for Actin 
(accession No. AB010922.1), F: TTGAATCCCAAGGCGAATAG and R: TGCGACCACTGGCATAAAG; for CsTUA (accession No. AJ715498.1), F: 5'-TTGTTCCTGGAGGCGATCTT-3' and R: 5' ACAAATGCGCGCTTAGCATA-3'. For CsGR (accession No. NM_001308836.1): F: $5^{\prime}$ GATATGAGAGCCGTGGTTGC-3' and 5'- AGTCGCAAACAACAC AGCAT-3'; for CsGAPDH (accession No. NM_001305758.1), 5'- TGACGA GTCCATCATCAGCAATGC-3' and 5'CAATGTTGAGTGCAGCAGCTCTTG-3'. The expression analyses were conducted three times independently.

\subsection{Statistical Analysis}

The statistical analyses was analyzed using the Statistical Package for Social Sciences for Windows (version 13.00; SPSS, Inc., Chicago, IC, United States) and statistical differences were analyzed through Duncan's multiple range test $(p<0.05)$. In the analysis of variance (ANOVA), results were expressed as the mean values $\pm \mathrm{SE}$ from three independent replicates.

\section{Conclusion}

Taken together, the evidence presented in this study showed that there are a series of $S$-nitrosylated proteins during NO-induction of the development of adventitious roots, which highlights the effect of NO-based posttranslational modification on regulating the development of adventitious roots in cucumber. Moreover, differential $S$-nitrosylation of key proteins regulated various pathways during adventitious rooting (Figure 6). Thus, our work demonstrated that $S$-nitrosylation process is an essential modulator during adventitious rooting of cucumber. Further work should focus on deciphering the function of such $S$-nitrosylated proteins on affecting adventitious root development. Therefore, corresponding genetic and proteomic evidences should be provided to further investigate mechanisms.

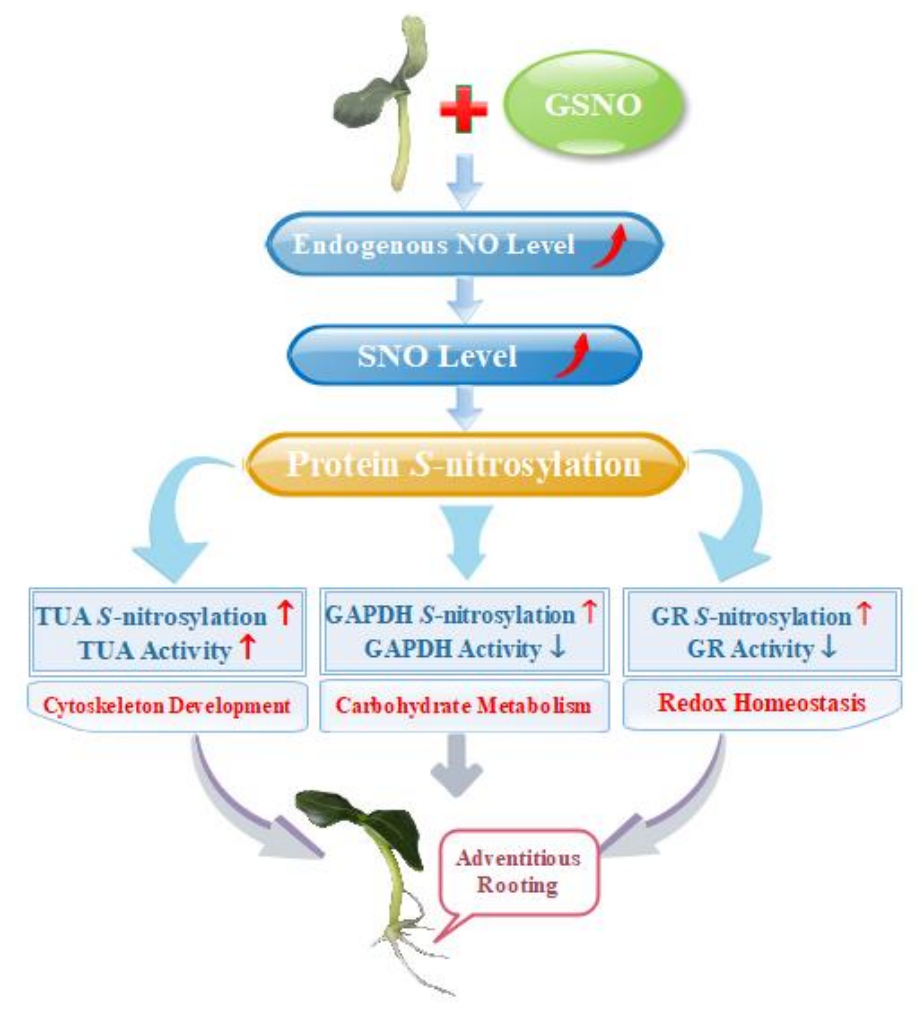

Figure 6. Schematic model of NO-induced S-nitrosylation during adventitious rooting in cucumber. NO-enhanced endogenous NO concentration and SNO levels, which triggers $S$-nitrosylation of proteins to induce adventitious root development. Differential S-nitrosylation of TUA, GAPDH and GR might regulate various pathways during NO-promoted the development of adventitious roots. The increase is indicated by the red arrow. The decrease is indicated by the blue arrow. 
Author Contributions: Conceptualization, J.Y.(Jihua Yu) and W.L.; Formal analysis, L.N., J.Y.(Jian Yu), J.L. and L.H.; Funding acquisition, J.Y.(Jihua Yu); Investigation, L.N., J.Y.(Jian Yu) and Y.W.; Methodology, L.N., W.L. and X.X.; Project administration, J.Y.(Jihua Yu) and J.X.; Resources, J.Y.(Jihua Yu); Supervision, J.Y.(Jihua Yu), W.L. and J.X.; Writing - original draft, L.N.; Writing - review \& editing, J.Y.(Jihua Yu) and W.L.

Funding: This work was supported by the National key research and development projects (2018YFD0201205), the National Natural Science Foundation of China (No. 31660584), China Agriculture Research System (CARS-23-C-07), Gansu Provience Science and Technology Key Project Fund (No.17ZD2NA015) and Natural Science Foundation of Gansu References Province, China (1610RJZA098).

Conflicts of Interest: The authors declare no conflict of interest. Declare conflicts of interest or state "The authors declare no conflict of interest." Authors must identify and declare any personal circumstances or interest that may be perceived as inappropriately influencing the representation or interpretation of reported research results. Any role of the funders in the design of the study; in the collection, analyses or interpretation of data; in the writing of the manuscript, or in the decision to publish the results must be declared in this section. If there is no role, please state "The funders had no role in the design of the study; in the collection, analyses, or interpretation of data; in the writing of the manuscript, or in the decision to publish the results".

\section{References}

1. Skiba, U.; Smith, K. Nitrification and denitrification as sources of nitric oxide and nitrous oxide in a sandy loam soil. Soil Biol. Biochem. 1993, 25, 1527-1536. [CrossRef]

2. Rockel, P.; Strube, F.; Rockel, A.; Wildt, J.; Kaiser, W.M. Regulation of nitric oxide (NO) production by plant nitrate reductase in vivo and in vitro. J. Exp. Bot. 2002, 53, 103-110. [CrossRef] [PubMed]

3. Corpas, F.; Barroso, J. Nitric oxide synthase-like activity in higher plants. Nitric Oxide-Biol. Chem. 2017, 68, 5-6. [CrossRef] [PubMed]

4. Niu, L.; Liao, W. Hydrogen Peroxide Signaling in Plant Development and Abiotic Responses: Crosstalk with Nitric Oxide and Calcium. Front. Plant Sci. 2016, 7. [CrossRef]

5. He, H.; Huang, W.; Oo, T.L.; Gu, M.; He, L.-F. Nitric oxide inhibits aluminum-induced programmed cell death in peanut (Arachis hypoganea L.) root tips. J. Hazard. Mater. 2017, 333, 285-292. [CrossRef]

6. Akram, N.A.; Iqbal, M.; Muhammad, A.; Ashraf, M.; Al-Qurainy, F.; Shafiq, S. Aminolevulinic acid and nitric oxide regulate oxidative defense and secondary metabolisms in canola (Brassica napus L.) under drought stress. Protoplasma 2018, 255, 163-174. [CrossRef]

7. Alamri, S.A.; Siddiqui, M.H.; Al-Khaishany, M.Y.; Khan, M.N.; Ali, H.M.; Alakeel, K.A. Nitric oxide-mediated cross-talk of proline and heat shock proteins induce thermotolerance in Vicia faba L. Environ. Exp. Bot. 2019, 161, 290-302. [CrossRef]

8. Neill, S.J.; Desikan, R.; Hancock, J.T. Nitric oxide signalling in plants. New Phytol. 2003, 159, 11-35. [CrossRef]

9. Pagnussat, G.C.; Lanteri, M.L.; Lamattina, L. Nitric oxide and cyclic GMP are messengers in the indole acetic acid-induced adventitious rooting process. Plant. Physiol. 2003, 132, 1241-1248. [CrossRef]

10. Liao, W.; Xiao, H.; Zhang, M. Role and relationship of nitric oxide and hydrogen peroxide in adventitious root development of marigold. Acta Physiol. Plant. 2009, 31, 1279. [CrossRef]

11. Astier, J.; Rasul, S.; Koen, E.; Manzoor, H.; Besson-Bard, A.; Lamotte, O.; Jeandroz, S.; Durner, J.; Lindermayr, C.; Wendehenne, D. S-nitrosylation: An emerging post-translational protein modification in plants. Plant Sci. 2011, 181, 527-533. [CrossRef] [PubMed]

12. Gaston, B.M.; Carver, J.; Doctor, A.; Palmer, L.A. S-nitrosylation signaling in cell biology. Mol. Interv. 2003, 3, 253-263. [CrossRef] [PubMed]

13. Hess, D.T.; Stamler, J.S. Regulation by S-nitrosylation of protein post-translational modification. J. Biol. Chem. 2012, 287, 4411-4418. [CrossRef]

14. Astier, J.; Kulik, A.; Koen, E.; Besson-Bard, A.; Bourque, S.; Jeandroz, S.; Lamotte, O.; Wendehenne, D. Protein S-nitrosylation: what's going on in plants? Free Radic. Biol. Med. 2012, 53, 1101-1110. [CrossRef] [PubMed]

15. Lamotte, O.; Bertoldo, J.B.; Besson-Bard, A.; Rosnoblet, C.; Aimé, S.; Hichami, S.; Terenzi, H.; Wendehenne, D. Protein S-nitrosylation: Specificity and identification strategies in plants. Front. Chem. 2015, 2, 114. [CrossRef]

16. Feng, J.; Chen, L.; Zuo, J. Protein S-nitrosylation in plants: Current progresses and challenges. J. Integr. Plant Biol. 2019. [CrossRef]

17. Aebersold, R.; Mann, M. Mass spectrometry-based proteomics. Nature 2003, 422, 198. [CrossRef] 
18. Hu, J.; Huang, X.; Chen, L.; Sun, X.; Lu, C.; Zhang, L.; Wang, Y.; Zuo, J. Site-specific nitrosoproteomic identification of endogenously $S$-nitrosylated proteins in Arabidopsis. Plant Physiol. 2015, 167, 1731-1746. [CrossRef]

19. Lindermayr, C.; Saalbach, G.; Durner, J. Proteomic identification of S-nitrosylated proteins in Arabidopsis. Plant Physiol. 2005, 137, 921-930. [CrossRef]

20. Morisse, S.; Zaffagnini, M.; Gao, X.-H.; Lemaire, S.D.; Marchand, C.H. Insight into protein S-nitrosylation in Chlamydomonas reinhardtii. Redox Signal. 2014, 21, 1271-1284. [CrossRef]

21. de Pinto, M.C.; Locato, V.; Sgobba, A.; del Carmen Romero-Puertas, M.; Gadaleta, C.; Delledonne, M.; De Gara, L. S-nitrosylation of ascorbate peroxidase is part of programmed cell death signaling in tobacco Bright Yellow-2 cells. Plant Physiol. 2013, 163, 1766-1775. [CrossRef] [PubMed]

22. Correa-Aragunde, N.; Foresi, N.; Lamattina, L. Nitric oxide is a ubiquitous signal for maintaining redox balance in plant cells: Regulation of ascorbate peroxidase as a case study. J. Exp. Bot. 2015, 66, 2913-2921. [CrossRef] [PubMed]

23. Yun, B.-W.; Feechan, A.; Yin, M.; Saidi, N.B.; Le Bihan, T.; Yu, M.; Moore, J.W.; Kang, J.-G.; Kwon, E.; Spoel, S.H. S-nitrosylation of NADPH oxidase regulates cell death in plant immunity. Nature 2011, 478, 264. [CrossRef] [PubMed]

24. Lombardo, M.C.; Lamattina, L. Abscisic acid and nitric oxide modulate cytoskeleton organization, root hair growth and ectopic hair formation in Arabidopsis. Nitric Oxide 2018, 80, 89-97. [CrossRef] [PubMed]

25. Yu, Q.-X.; Ahammed, G.J.; Zhou, Y.-H.; Shi, K.; Zhou, J.; Yu, Y.; Yu, J.-Q.; Xia, X.-J. Nitric oxide is involved in the oxytetracycline-induced suppression of root growth through inhibiting hydrogen peroxide accumulation in the root meristem. Sci. Rep. 2017, 7, 43096. [CrossRef] [PubMed]

26. Liu, M.; Liu, X.X.; He, X.L.; Liu, L.J.; Wu, H.; Tang, C.X.; Zhang, Y.S.; Jin, C.W. Ethylene and nitric oxide interact to regulate the magnesium deficiency-induced root hair development in Arabidopsis. New Phytol. 2017, 213, 1242-1256. [CrossRef]

27. Yuan, H.M.; Huang, X. Inhibition of root meristem growth by cadmium involves nitric oxide-mediated repression of auxin accumulation and signalling in Arabidopsis. Plant Cell Environ. 2016, 39, 120-135. [CrossRef]

28. Lanteri, M.L.; Laxalt, A.M.; Lamattina, L. Nitric oxide triggers phosphatidic acid accumulation via phospholipase D during auxin-induced adventitious root formation in cucumber. Plant Physiol. 2008, 147, 188-198. [CrossRef]

29. Jin, X.; Liao, W.B.; Yu, J.H.; Ren, P.J.; Dawuda, M.; Wang, M.; Niu, L.J.; Li, X.P.; Xu, X.T. Nitric oxide is involved in ethylene-induced adventitious rooting in marigold (Tagetes erecta L.). Sci. Hortic. 2017, 97, 620-631. [CrossRef]

30. Niu, L.; Yu, J.; Liao, W.; Yu, J.; Zhang, M.; Dawuda, M.M. Calcium and calmodulin are involved in Nitric Oxide-induced adventitious rooting of cucumber under simulated osmotic stress. Front. Plant Sci. 2017, 8, 1684. [CrossRef]

31. Feng, J.; Wang, C.; Chen, Q.; Chen, H.; Ren, B.; Li, X.; Zuo, J. S-nitrosylation of phosphotransfer proteins represses cytokinin signaling. Nat. Commun. 2013, 4, 1529. [CrossRef] [PubMed]

32. Wang, J.; Wang, Y.; Lv, Q.; Wang, L.; Du, J.; Bao, F.; He, Y.-K. Nitric oxide modifies root growth by $S$-nitrosylation of plastidial glyceraldehyde-3-phosphate dehydrogenase. Biochem. Biophys. Res. Commun. 2017, 488, 88-94. [CrossRef] [PubMed]

33. Terrile, M.C.; París, R.; Calderón-Villalobos, L.I.; Iglesias, M.J.; Lamattina, L.; Estelle, M.; Casalongué, C.A. Nitric oxide influences auxin signaling through $S$-nitrosylation of the Arabidopsis TRANSPORT INHIBITOR RESPONSE 1 auxin receptor. Plant J. 2012, 70, 492-500. [CrossRef] [PubMed]

34. Feechan, A.; Kwon, E.; Yun, B.-W.; Wang, Y.; Pallas, J.A.; Loake, G.J. A central role for S-nitrosothiols in plant disease resistance. Proc. Natl. Acad. Sci. USA 2005, 102, 8054-8059. [CrossRef]

35. Frungillo, L.; Skelly, M.J.; Loake, G.J.; Spoel, S.H.; Salgado, I. S-nitrosothiols regulate nitric oxide production and storage in plants through the nitrogen assimilation pathway. Nat. Commun. 2014, 5, 5401. [CrossRef]

36. Lin, A.; Wang, Y.; Tang, J.; Xue, P.; Li, C.; Liu, L.; Hu, B.; Yang, F.; Loake, G.J.; Chu, C. Nitric oxide and protein $S$-nitrosylation are integral to hydrogen peroxide-induced leaf cell death in rice. Plant Physiol. 2012, 158, 451-464. [CrossRef] 
37. Rapaka, V.K.; Bessler, B.; Schreiner, M.; Druege, U. Interplay between initial carbohydrate availability, current photosynthesis, and adventitious root formation in Pelargonium cuttings. Plant Sci. 2005, 168, 1547-1560. [CrossRef]

38. Akram, M. Citric acid cycle and role of its intermediates in metabolism. Cell Biochem. Biophys. 2014, 68, 475-478. [CrossRef]

39. Nick, P. Signals, motors, morphogenesis-the cytoskeleton in plant development. Plant Biol. 1999, 1, $169-179$. [CrossRef]

40. Jaffrey, S.R.; Erdjument-Bromage, H.; Ferris, C.D.; Tempst, P.; Snyder, S.H. Protein S-nitrosylation: A physiological signal for neuronal nitric oxide. Nat. Cell Biol. 2001, 3, 193. [CrossRef]

41. Yemets, A.I.; Krasylenko, Y.A.; Lytvyn, D.I.; Sheremet, Y.A.; Blume, Y.B. Nitric oxide signalling via cytoskeleton in plants. Plant Sci. 2011, 181, 545-554. [CrossRef] [PubMed]

42. Shenton, D.; Grant, C.M. Protein S-thiolation targets glycolysis and protein synthesis in response to oxidative stress in the yeast Saccharomyces cerevisiae. Biochem. J. 2003, 374, 513-519. [CrossRef] [PubMed]

43. Sen, N.; Hara, M.R.; Kornberg, M.D.; Cascio, M.B.; Bae, B.-I.; Shahani, N.; Thomas, B.; Dawson, T.M.; Dawson, V.L.; Snyder, S.H. Nitric oxide-induced nuclear GAPDH activates p300/CBP and mediates apoptosis. Nat. Cell Biol. 2008, 10, 866. [CrossRef] [PubMed]

44. Kornberg, M.D.; Sen, N.; Hara, M.R.; Juluri, K.R.; Nguyen, J.V.K.; Snowman, A.M.; Law, L.; Hester, L.D.; Snyder, S.H. GAPDH mediates nitrosylation of nuclear proteins. Nat. Cell Biol. 2010, 12, 1094. [CrossRef]

45. Meyer, A.J. The integration of glutathione homeostasis and redox signaling. J. Plant Physiol. 2008, 165, 1390-1403. [CrossRef]

46. Bao, Y.; Kost, B.; Chua, N.H. Reduced expression of $\alpha$-tubulin genes in Arabidopsis thaliana specifically affects root growth and morphology, root hair development and root gravitropism. Plant J. 2001, 28, 145-157. [CrossRef]

47. Zhu, Y.; Liao, W.; Niu, L.; Wang, M.; Ma, Z. Nitric oxide is involved in hydrogen gas-induced cell cycle activation during adventitious root formation in cucumber. BMC Plant Biol. 2016, 16, 146. [CrossRef]

48. Begara-Morales, J.C.; Sánchez-Calvo, B.; Chaki, M.; Mata-Pérez, C.; Valderrama, R.; Padilla, M.N.; López-Jaramillo, J.; Luque, F.; Francisco, J.C.; Barroso, J.B. Differential molecular response of monodehydroascorbate reductase and glutathione reductase by nitration and S-nitrosylation. J. Exp. Bot. 2015, 66, 5983-5996. [CrossRef]

49. Lorbiecke, R.; Sauter, M. Adventitious root growth and cell-cycle induction in deepwater rice. Plant Physiol. 1999, 119, 21-30. [CrossRef]

50. Beltrán, B.; Orsi, A.; Clementi, E.; Moncada, S. Oxidative stress and S-nitrosylation of proteins in cells. Br. J. Pharmacol. 2000, 129, 953-960. [CrossRef]

51. Graziano, M.; Lamattina, L. Nitric oxide accumulation is required for molecular and physiological responses to iron deficiency in tomato roots. Plant J. 2007, 52, 949-960. [CrossRef]

52. Xuan, W.; Xu, S.; Li, M.; Han, B.; Zhang, B.; Zhang, J.; Lin, Y.; Huang, J.; Shen, W.; Cui, J. Nitric oxide is involved in hemin-induced cucumber adventitious rooting process. J. Plant Physiol. 2012, 169, 1032-1039. [CrossRef]

53. Durner, J.; Wendehenne, D.; Klessig, D.F. Defense gene induction in tobacco by nitric oxide, cyclic GMP, and cyclic ADP-ribose. Proc. Natl. Acad. Sci. USA 1998, 95, 10328-10333. [CrossRef]

54. Jaffrey, S.R.; Snyder, S.H. The biotin switch method for the detection of S-nitrosylated proteins. Sci. STKE 2001, 86, pl1. [CrossRef]

55. Foyer, C.H.; Halliwell, B. The presence of glutathione and glutathione reductase in chloroplasts: A proposed role in ascorbic acid metabolism. Planta 1976, 133, 21-25. [CrossRef]

56. Piattoni, C.; Guerrero, S.; Iglesias, A. A differential redox regulation of the pathways metabolizing glyceraldehyde-3-phosphate tunes the production of reducing power in the cytosol of plant cells. Int. J. Mol. Sci. 2013, 14, 8073-8092. [CrossRef]

57. Zhao, Y.; Liu, W.; Xu, Y.-P.; Cao, J.-Y.; Braam, J.; Cai, X.-Z. Genome-wide identification and functional analyses of calmodulin genes in Solanaceous species. BMC Plant Biol. 2013, 13, 70. [CrossRef]

(C) 2019 by the authors. Licensee MDPI, Basel, Switzerland. This article is an open access article distributed under the terms and conditions of the Creative Commons Attribution (CC BY) license (http://creativecommons.org/licenses/by/4.0/). 